\title{
Joint cross-layer resource allocation and interference avoidance with QoS support for cognitive radio systems
}

\author{
Hailan Peng ${ }^{*}$ and Takeo Fujii
}

\begin{abstract}
In this article, we study the coexistence and optimization of a centralized orthogonal frequency-division multiple access-based multiuser cognitive radio (MCR) system which coexists with a cellular primary system (PS). Two different spectrum sharing methods, i.e., the spectrum underlay/overlay, are utilized for different coexistent frameworks, in which, the sharing method is adapted to one of them based on the distance between two base stations and the interference limit in the PS. We consider a PS-assistance-based coexistent architecture and propose a joint power control and interference management method to avoid unacceptable interference to primary users (PU). Firstly, the relationship between power limits at secondary users (SU) and interference margin at PUs can be obtained. Then, to provide the SUs with satisfactory quality of service (QoS), and optimize the sum rate of the MCR system as well, a constrained two-variable nonlinear optimization problem (OP) is formulated. We solve this OP by (i) simplifying the QoS constraints from medium access control-layer to physical-layer based on a crosslayer approximation; and (ii) using the Lagrangian duality based technique to solve the simplified OP, and iterative water-filling is implemented to find the optimal power and subcarrier allocation. Simulation results show that, compared to the conventional designs, our algorithm achieves significant higher throughput and can guarantee the required signal to interference plus noise ratio of the PUs and the QoS of the SUs well. Moreover, compared to the spectrum overlay sharing method, the spectrum underlay \& overlay can provide substantial higher spectrum efficiency.
\end{abstract}

Keywords: multiuser cognitive radio, cross-layer design, orthogonal frequency-division multiple access (OFDMA), QoS, Lagrangian duality optimization

\section{Introduction}

Due to the rapid growth of wireless communications, the problem of spectrum shortage has become much severer. The report from the Federal Communications Commission (FCC) has shown that most of the licensed spectrum is currently under-utilized [1]. Cognitive radio (CR) is a promising technology that can alleviate the severe spectrum shortage problem by making it possible for secondary (unlicensed) users (SU) to share frequency bands with primary (licensed) users (PU) in some geographical location $[2,3]$. The SUs equipped with CR can sense and learn their surrounding environment to find a

\footnotetext{
* Correspondence: penghailan@awcc.uec.ac.jp Advanced Wireless Communication Research Center, The University of Electro-Communications, 1-5-1 Chofugaoka, Chofu-shi, Tokyo 182-8585, Japan
}

spectrum band for opportunistic communication using either spectrum overlay (non-active PU bands sharing) or spectrum underlay (whole PU bands sharing).

Even though the basic idea of CR is simple, the efficient design of $\mathrm{CR}$ systems imposes the new challenges compared to conventional wireless systems. In a CR system, the basic philosophy is to allow universal maximization of the spectrum utilization, and the utilization by the SUs cannot degrade the service in a primary system (PS). To flexibly implement spectrum sharing between the PUs and the SUs and enhance the spectrum efficiency, a dynamic resource allocation (DRA) for multiple SUs is required. Therefore, the orthogonal frequency division multiple-access (OFDMA) technique is an attractive candidate for such a flexible multiuser CR system [4]. 
Various resource allocation (RA) methods have been developed for OFDM-based CR systems. In [5,6], the fair RA of subcarrier, bit, and power in physical layer (PHY) to maximize the system throughput while guaranteeing the interference power limited is studied for OFDM-based CR systems. However, these algorithms cannot dynamically adjust their rate requirement to different SUs. Moreover, in [6], the authors assume that the PS and the CR system are both OFDM-based systems, where it is impractical that the PS always uses OFDM. Currently, to the best of authors' knowledge, there are few studies on quality of service (QoS) support in OFDMA-based CR systems. The QoS designs in $[7,8]$ for CR systems only considered non-real-time applications. Up to now, the study on the spectrum underlay sharing is also sparse. A non-active PU bands access based spectrum overlay sharing was considered in $[9,10]$ for RA in OFDM-based CR systems.

Despite the above contributions, the coexistence and optimization of a multiuser cognitive radio (MCR) system taking into account the mutual interference (MI) [11], QoS support and the different spectrum sharing schemes still have not been well studied. Several technical difficulties are involved. First, the CR-system/PS coexistence involves the MI (the SU-to-PU interference as well as the PU-to-SU interference), which is computationally complex and inaccurate. Furthermore, this should be obtained using very limited information. The $\mathrm{CR}$ system has to maximize the sum rate of all SUs, and at the same time make sure the SU-to-PU interference at each PU receiver does not exceed a limit. Second, to account for the MI, limited transmit power and satisfactory QoS, a large number of constraints are involved in the optimization procedure. Simplified and fast update algorithms are needed.

For the CR-system/PS coexistence, we consider a novel infrastructure-based dynamic system architecture, in which the CR system can be either independent or overlapped with the PS cell. Moreover, a PS-assistancebased joint spectrum underlay/overlay method is proposed for the spectrum sharing and realtime SU-to-PU interference control. First, a primary base station (PBS) determines the interference limits that can be accepted at each PU receiver based on its target performance, such as predefined signal to interference plus noise ratio (SINR), system outage probability, and so on. Then, the PBS broadcasts the interference limits on its occupied subbands and pilot signals for SU-to-PU interference channel estimation. According to the interference limits and geographic location of the CR system, a cognitive base station (CBS) decides available spectrum resources in the CR system and utilizes adaptive power control $[12,13]$ to limit the SU-to-PU interference.
For the DRA, we propose a Lagrangian duality-based optimization framework under transmit power and QoS constraints for downlink transmissions. Our considered scenario can be modeled as a constrained two-variable nonlinear optimization problem (OP). In order to solve the problem and achieve our objectives, we develop near-optimal and low-complexity approaches. Firstly, based on the transmit power of the CBS and the interference limits of PU receivers, a joint power control and interference avoidance method has been analyzed to simplify the constraints and guarantee the performance in the PS with priority. Then, a cross-layer design and the Lagrangian dual problem method have been considered to transform the QoS requirements in medium access control (MAC) layer to PHY-layer, so as to provide QoS support for the SUs during each scheduling time. Finally, iterative water-filling (IWF) algorithm has been implemented to solve the near-optimal and lowcomplexity problem of the system. Then, three subproblems have been deduced to get the solutions.

The rest of this article is organized as follows. In Section 2, the system models and related assumptions are described, which include the system architecture, the wireless propagation model, and the interference signal of the SUs. In Section 3, the constraints and the constrained nonlinear OP have been formulated. In Section 4 , the joint cross-layer optimization is elaborately considered. Simulation and numerical results have been shown in Section 5. The results show that the joint cross-layer design has significant improvement compared to two conventional designs. Compared to the spectrum overlay sharing, the spectrum underlay sharing can provide a substantial performance improvement due to the higher spectrum efficiency. Finally, conclusions are drawn in Section 6.

\section{System model and interference of SUs 2.1 OFDMA-based MCR system}

We consider an OFDMA-based MCR system with $K$ SUs, and a CBS as the controller to share the spectrum with a PS, which is also an infrastructure-based cellular system, with one PBS and $N$ PUs.

In practical applications, it is possible that uplink transmit power of PUs is too small, then, the MCR system cannot access primary bands in order to protect the PS. Therefore, with considering the feasibility of the coexistent architecture, we assume that, in the PS, uplink and downlink transmissions use the time-division duplex (TDD) mode, meanwhile, in the MCR system, the frequency-division duplexing (FDD) is employed. Hence, in the worst case, both uplinks and downlinks of the MCR system can access primary bands when the PS is on downlink transmissions. 
In this article, we consider downlink transmissions in both the CR system and the PS at the same time. The whole spectrum, which is originally licensed to PUs, is divided into $M$ subcarriers in the CR system. Active subbands allocated to the PUs and OFDM subcarriers of the SUs have been shown in Figure 1, where, the bandwidth of subband $n$ is denoted by $B_{n}$, which is allocated to $\mathrm{PU} n$, and $\Delta f$ is the subcarrier spacing of the CR system. The time slot duration in the CR system is equal to one OFDM symbol period $T_{s}$ and the subcarriers are modeled in discrete time with the time-varying gain. The set of the SUs, the PUs, the subcarriers and the time slots are denoted by $\mathcal{K}=\{1, \ldots, K\}, \mathcal{N}=\{1, \ldots, N\}, \mathcal{M}=\{1, \ldots, M\}, \quad$ and $\mathcal{T}=\{1, \ldots, T\}$, respectively.

\subsection{PHY and propagation model}

For all the links in the MCR system, the channels are subject to frequency selective fading. The channel gain is given by:

$$
G_{k, m, t}=g_{k, m, t} \cdot \mu\left(d_{k} / d_{0}\right)^{-\alpha}, \quad \forall k \in \mathcal{K}, \quad \forall m \in \mathcal{M}, \quad \forall t \in \mathcal{T}
$$

where $\sqrt{g_{k, m, t}}$ is the small-scale fading being modeled as a Rayleigh distribution. $\mu$ is the free-space factor of the channel gain, which can be calculated from $\mu=G_{s}\left(\frac{\lambda}{4 \pi d_{0}}\right)^{2}$, where, $G_{s}$ denotes the transmit and receive antenna gain, $\lambda$ is the wave length, $d_{0}$ is a reference distance, set to be $d_{0}=10 \mathrm{~m} \mathrm{[14]} . d_{k}$ denotes the distance between the CBS and SU $k . \alpha$ is the path loss exponent. For any link, the power gains $G_{k, m, t}$ are independent identical distribution (i.i.d.) random variables. Furthermore, we assume that the channel is block fading, i.e., $g_{k, m, t}$ is fixed during each time slot, which is much longer than the total duration of information collecting and reporting.

\subsection{Interference to SUs}

In the coexistent system, we consider two kinds of MI: the interference from the PBS to SUs and that from the CBS to PUs. The first interference is discussed here. The second one will be introduced in Section 3.1.

In the CR system, we assume that the interference from primary transmitters to SUs can be measured properly. Therefore, after the interference limits and the pilot signals from the PBS were collected, the channel information between two systems can be known at the CBS.

According to Figure 1, the interference power generated by primary transmitters (i.e., the PBS on downlink, or PUs on uplink) to SU $k$ at subcarrier $m$ and time slot $t$ can be given by

$$
I_{k, m, t}= \begin{cases}P_{p}^{(d)} G_{k, m, t}^{p s} \int_{(m-1) \Delta f}^{m \Delta f} \Phi_{p}(f) d f, \quad \text { if downlinks in the PS } \\ \sum_{n=1}^{N} P_{n}^{(u)} G_{k, m, t}^{n} \int_{(m-1) \Delta f}^{m \Delta f} \Phi_{n}(f) d f, \text { if uplinks in the PS }\end{cases}
$$

where, $P_{p}^{(d)}$ is the downlink transmit power at the PBS, $G_{k, m, t}^{\mathrm{ps}}$ is the power gain from the PBS to SU $k, \Phi_{p}$ $(f)$ is the equivalent baseband power spectral density (PSD) of the PBS signal when the transmit power is normalized to one watt. $P_{n}^{(u)}$ is the uplink transmit power at $\mathrm{PU} n, G_{k, m, t}^{n}$ is the power gain from $\mathrm{PU} n$ to $\mathrm{SU} k$, $\Phi_{n}(f)$ is the normalized equivalent baseband PSD of the PU $n$ signal.

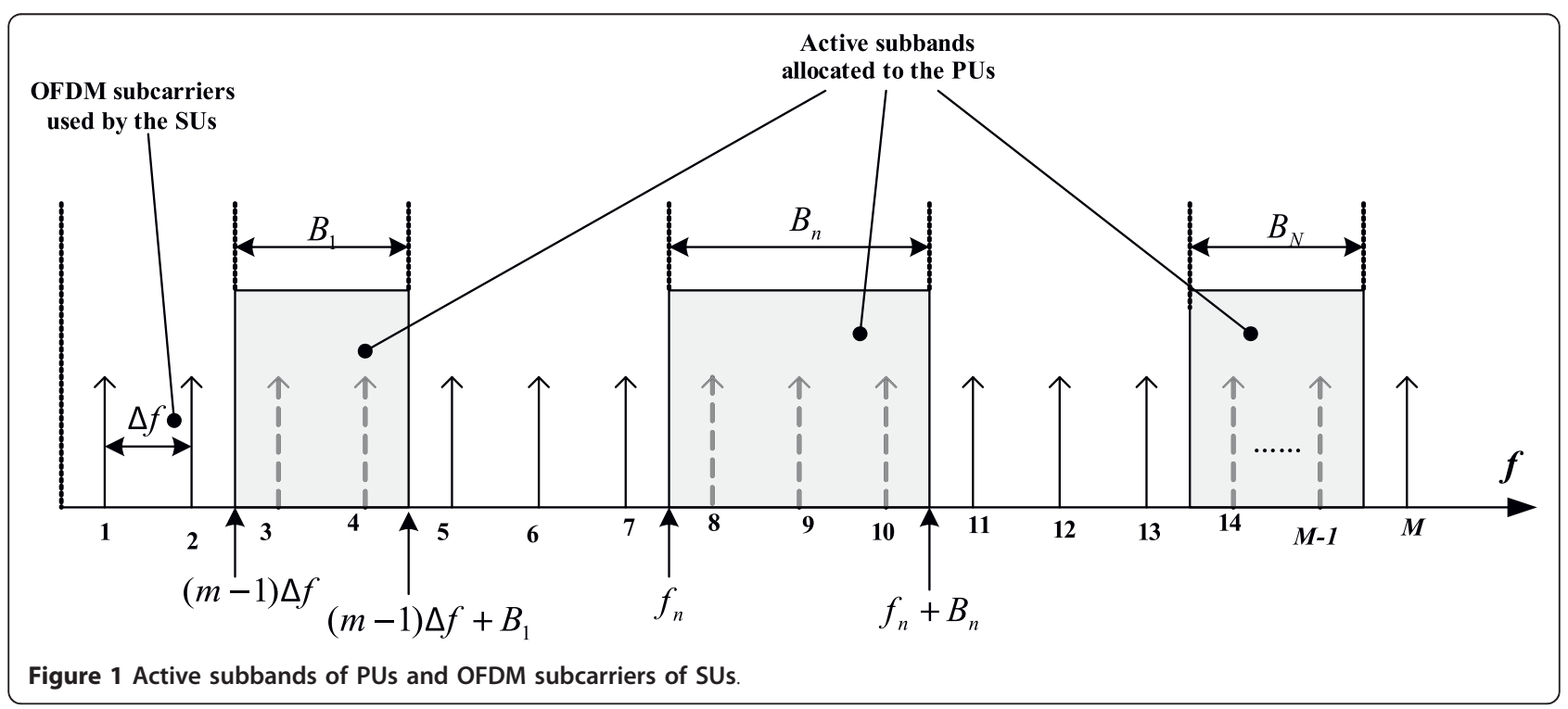


We now make further assumptions about channel gain information. We assume that the transmit power and PSD $\Phi_{p}(f)$ and $\Phi_{n}(f)$ are known to SUs, based on these, the SUs can estimate the mean channel gains from the primary transmitters to themselves. Due to the reciprocal characteristic of the wireless channel, the mean channel gains from the SUs to the primary transmitters would be equal to these values. Similarly, the mean channel gains from the CBS to primary transmitters also can be estimated.

\section{Constraints and problem definition}

\subsection{Power limits for SUs}

In this article, we use a predefined SINR value $\gamma_{p}$ and an interference violation probability $\delta^{(p)}$ together as the primary target matrix. First, the received SINR at each PU must be no less than the predefined value $\gamma_{p}$. Let $\gamma_{n, t}$ denote the SINR experienced by PU $n$ at subband $n$. Therefore, we must have:

$$
\gamma_{n, t}=\frac{P_{n, t}^{\mathrm{PBS}} G_{n, t}^{p}}{N_{0}+I_{n}} \geq \gamma_{p}, \quad \forall n \in \mathcal{N}
$$

where $P_{n, t}^{\mathrm{PBS}}$ is the transmit power from the PBS to PU $n$. $G_{n, t}^{p}$ is the channel gain between the PBS and PU $n$, which is frequency selective over subband $n . N_{0}$ is the complex Gaussian noise power. $I_{n}$ is the SU-to-PU interference from the CBS to PU $n$. According to (3), the interference limit $I_{n}^{\max }$ of PU $n$ can be obtained as following:

$$
I_{n}^{\max }=\frac{P_{n, t}^{\mathrm{PBS}} G_{n, t}^{p}}{\gamma_{p}}-N_{0}
$$

where, the set $\left\{I_{n}^{\max }, n \in \mathcal{N}\right\}$ is the interference power limits at the PUs.

Assume that $B_{n}$ is a multiple of $\Delta f$, which is from frequency $f_{n}$ to frequency $f_{n}+B_{n}$, as shown in Figure 1. Let $x$ be the beginning subcarrier index of subband $n$, so $f_{n}$ $=(x-1) \Delta f$. The SU-to-PU interference $I_{n}$, which should be no larger than the interference limit $I_{n}^{\max }$, can be given by:

$$
I_{n}=\sum_{m=x}^{S+x} P_{k, m, t} G_{n, m, t}^{\mathrm{sp}} \int_{(m-1) \Delta f}^{m \Delta f} \Phi_{s}(f) d f \leq I_{n}^{\max }
$$

where, $S$ is the total secondary subcarrier number by which PU subband $n$ is affected, $P_{k, m, t}$ is the allocated transmit power from the CBS to SU $k$ on subcarrier $m$ and time slot $t, G_{n, m, t}^{\mathrm{sp}}$ is the channel gain from the CBS to PU $n$ on subcarrier $m . \Phi_{s}(f)$ is the normalized equivalent baseband PSD of the secondary OFDM signal. Here, we only consider the main lobe power of the OFDM signal, because the interference power caused by the side lobes of the OFDM signal is very low, only $4.922 \%$ of the transmit power without multiplying the path loss [11]. Hence, we can assume $P_{k, m, t} \int_{(m-1) \Delta f}^{m \Delta f} \Phi_{s}(f) d f \approx P_{k, m, t}$ for simplicity. Moreover, as long as the interference power on any subcarrier $m$ has been controlled no larger than the average limit $I_{n}^{\max } / S$, the predefined SINR at each PU can be guaranteed. Let $I_{m}^{\mathrm{lim}}=I_{n}^{\max } / S$ denote the interference limit for subcarrier $m$. Therefore, Equation (5) can be rewritten as:

$$
I_{m}=P_{k, m, t} G_{n, m, t}^{\mathrm{sp}} \leq I_{m}^{\mathrm{lim}}
$$

where, $\left\{I_{m}^{\lim }=\left(I_{n}^{\max } / S\right)^{+}, n \in \mathcal{N}, m \in \mathcal{M}\right\}$ is a mapping of the interference limits from primary subband $n$ to $S$ secondary subcarriers, where, $(x)^{+}=\max (0, x)$.

In the CR-system, the adaptive power control is used to manage the interference from the CBS to PUs. Since the instantaneous interference level $I_{n}$ may exceed the tolerable limit $I_{n}^{\max }$, and violate the absolute interference constraint, we define the interference violation probability as $\operatorname{Pr}\left\{I_{n}>I_{n}^{\max }\right\}$, where, $\operatorname{Pr}\{A\}$ denotes the probability of event $\mathrm{A}$, which should be no larger than the value $\delta^{(p)}$. Therefore, considering the simplification from (5) to (6), there is a constraint on the interference violation probability $\delta^{(p)}$ as following:

$$
\operatorname{Pr}\left\{P_{k, m, t} G_{n, m, t}^{\mathrm{sp}}>I_{m}^{\lim }\right\} \leq \delta^{(p)}, \quad \forall m \in \mathcal{M}
$$

Similar to Equation (1), $G_{n, m, t}^{\mathrm{sp}}=g_{n, m, t}^{\mathrm{sp}} \cdot \mu\left(d_{n, t}^{\mathrm{sp}} / d_{0}\right)^{-\alpha}$, where $g_{n, m, t}^{\mathrm{sp}}$ is the small scale fading between the CBS and PU $n$ on subcarrier $m$, and has been characterized as a Rayleigh distribution with the probability density function (PDF) $f(x ; \sigma)=\frac{x}{\sigma^{2}} e^{\left(-x^{2} / 2 \sigma^{2}\right)}$ and the cumulative distribution function (CDF) $F(x)=1-e^{\left(-x^{2} / 2 \sigma^{2}\right)}$, where $x \in[0, \infty), \sigma>0 . d_{n, t}^{\mathrm{sp}}$ is the distance between the CBS and PU $n$.

From (7), we have the following proposition for power control at the CBS.

Propositon 1: At the CBS, the allocated power for each subcarrier should be controlled no larger than the following value:

$$
P_{m, t}^{\max }=\frac{I_{m}^{\lim }}{\mu\left(d_{n, t}^{\mathrm{sp}} / d_{0}\right)^{-\alpha} F^{-1}\left(1-\delta^{(p)}\right)}, \quad \forall m \in \mathcal{M}
$$


where $F^{-1}($.$) is the inverse function of the CDF of the$ Rayleigh distribution. Assume that location information of the PUs is available to the CBS where a RA algorithm is executed. A variety of location-awareness techniques are introduced in [15] and the references therein. The location-based primary protection and RA methods can be found in $[16,17]$. However, the methods proposed in $[16,17]$ cannot guarantee the QoS for each SU, and they ignored the small-scale fading totally.

Proof: Details are provided in Appendix 1.

Then, the interference constraint (6) for PUs can be replaced by the following power limit for SUs:

$$
P_{k, m, t} \leq P_{m, t}^{\max }, \quad \forall m \in \mathcal{M}
$$

where, $P_{m, t}^{\max }$ is the maximum power that can be allocated to subcarrier $m$. This power-limited access control method is based on the assumptions and system models in this article.

\subsection{QoS constraints for SUs}

At MAC layer, a cross-layer RA algorithm is proposed to support both real-time (RT) and non-real-time (NRT) services in the CR system. Assume that the first $i$ users from $K$ are with RT service, denoted by $\mathcal{I}=\{1, \ldots, i\}$; and the other $K-i$ users are with NRT service, denoted by $\mathcal{J}=\{i+1, \ldots, K\}$.

Real-time service is the services such as MPEG or streaming video or audio. It provides guarantees on throughput and latency, that is, each packet, which has a length of $l_{i}^{\mathrm{RT}}$, needs to be received by $\mathrm{SU} i$ within $d_{i}^{\mathrm{RT}}$ time slots after the packet has been transmitted.

Non-real-time service provides guarantees on throughput, can tolerate longer delays and is insensitive to delay jitter. So it is suitable for FTP applications. Its average data rate that the system needs to provide is $R_{j}^{\mathrm{NRT}}$.

The instantaneous rate for SU $k$ at subcarrier $m$ and time slot $t$ can be given by:

$$
R_{k, m, t}=\Delta f \log _{2}\left(1+\beta_{k, m, t} P_{k, m, t}\right), \quad \forall k \in \mathcal{K}
$$

where, $\beta_{k, m, t}=\frac{G_{k, m, t}}{N_{0}+I_{k, m, t}}, I_{k, m, t}$ is defined in Section 2.3, $P_{k, m, t}$ is limited by Equation (9). In order to provide satisfactory QoS for the SUs, there are following constraints for different services:

$$
\begin{aligned}
& t_{i}^{D}-t_{i}^{S} \leq d_{i}^{\mathrm{RT}}, \quad \forall i \in \mathcal{I} \\
& \sum_{t=1}^{d_{i}^{\mathrm{RT}}} \sum_{m=1}^{M} R_{i, m, t} \geq l_{i}^{\mathrm{RT}}, \quad \forall i \in \mathcal{I}, \quad m \in \mathcal{M}, \quad t \in \mathcal{T}
\end{aligned}
$$

$$
\bar{R}_{j}=\frac{1}{T} \sum_{t=1}^{T} \sum_{m=1}^{M} R_{j, m, t} \geq R_{j}^{\mathrm{NRT}}, \quad \forall j \in \mathcal{J}, \quad m \in \mathcal{M}, \quad t \in \mathcal{T}
$$

where, for RT SUs, $t_{i}^{D}$ is the arriving time slot when the data arrived at the destination (i.e., $\mathrm{SU} i$ ), and $t_{i}^{S}$ is the sending time slot from the source node (i.e., the $\mathrm{CBS}$ ). Inequality (11) indicates that the duration time slots from the CBS to SU $i$ should be no larger than the delay of RT SUs $d_{i}^{\mathrm{RT}}$. In (12), the packet length $l_{i}^{\mathrm{RT}}$ needs to be received by $\mathrm{SU} i$ within $d_{i}^{\mathrm{RT}}$ time slots, that is, Equation (12) is another form of (11). Equation (13) is the MAC-layer QoS constraint for NRT SUs, where, $\bar{R}_{j}$ is the average rate of $S U j$ from time Slot 1 to time slot $T$, and should be no smaller than $R_{j}^{\mathrm{NRT}}$.

\subsection{OP for the MCR system}

In this article, our objective is to maximize the system throughput under several constraints while ensuring that the RT services can be provided within their specified deadlines, as well as the average data rates for NRT can satisfy the requirements. Let $\mathcal{L}=\left\{L_{k, m}, t, k \in \mathcal{K}, m \in \mathcal{M}, t \in \mathcal{T}\right\}$ denote the allocation results of the continuous instantaneous $M$ subcarriers in the time slot $t$. Therefore, based on the above system models, the OP can be formulated as follows:

OP-1:

$$
\max \sum_{t=1}^{T} \sum_{m=1}^{M} \sum_{k=1}^{K} R_{k, m, t} L_{k, m, t}
$$

s.t. Equation (9), (11)-(13) and

$$
\begin{aligned}
& \sum_{k=1}^{K} \sum_{m=1}^{M} P_{k, m, t} \leq P_{0} \\
& \sum_{k=1}^{K} L_{k, m, t} \leq 1, \quad L_{k, m, t}=\{0,1\}
\end{aligned}
$$

$$
0 \leq R_{k, m, t} \leq R_{\max }
$$

The system utility function (14) is an OP, where, $T$ is the number of time slots considered in the allocation algorithm; $M$ is the total subcarrier number in each time slot. In (15), $P_{0}$ is the total allowed power in the CBS per time slot. Equation (16) is constraint to guarantee that there is only one connection on one subcarrier. To protect the PS better, besides the interference limits from the PBS, we consider a maximum link rate $R_{\max }$ to limit the transmit power from the CBS further. The (17) 
indicates that the actual link capacity on each subcarrier should be no more than $R_{\max }$, where, $R_{\max }=r_{\max } \Delta f$, $r_{\max }$ is the maximum number of bits that can be allowed per subcarrier.

\section{Joint cross-layer RA and interference avoidance with QoS support}

The OP-1 in Section 3.3 is difficult to solve since it involves an optimization over $T$ time slots, $M$ subcarriers, $K$ users, and with MAC-layer QoS requirements. The CBS should be able to allocate the subcarriers and power to $K$ SUs at the beginning of $T$ time slots based on the solution of OP-1, therefore, in OP-1, the knowledge of future channel gains (i.e., the channel gain at future time slots) is required. However, it is impossible for the CBS to obtain future channel information, so, we need to simplify the QoS requirements over $T$ time slots to one time slot. In the following section, we formulate problem OP-2, which is a power minimization problem to find the minimum transmit power that can guarantee the QoS requirements for SUs during $\tau$ time slots $(\tau$ $<T$ ), so as to transform the MAC-layer QoS constraints over $T$ time slots to less time slots.

4.1 Transform the MAC-layer QoS constraints to PHY-layer Consider the QoS requirements in $\tau$ time slots, Equations (11) and (12) can be rewritten as:

$$
\sum_{t=1}^{\tau} \sum_{m=1}^{M} R_{i, m, t} L_{i, m, t} \geq r_{i, \tau}^{\mathrm{Req}}, \quad \forall i \in \mathcal{I}, \quad \tau \in 1,2, \ldots, T, \quad \tau<d_{i}^{\mathrm{RT}}
$$

where, $\tau$ is the number of time slots considered in the RA algorithm, which is less than $T . r_{i, \tau}^{\text {Req }}$ is the packet length that needs to be transmitted in $\tau$ time slots for RT SU $i$.

Equation (13) can be rewritten as:

$$
\sum_{t=1}^{\tau} \sum_{m=1}^{M} R_{j, m, t} L_{j, m, t} \geq r_{j, \tau}^{\mathrm{Req}}, \quad \forall j \in \mathcal{J}, \quad \tau \in 1,2, \ldots, T
$$

where, $r_{i, \tau}^{\text {Req }}$ is the total bit rate that needs to be transmitted in $\tau$ time slots for NRT SU $j$.

Combining (18) and (19) together, the QoS requirements in (11)-(13) can be rewritten as a rate constraint during $\tau$ time slots, that is:

$$
\sum_{t=1}^{\tau} \sum_{m=1}^{M} R_{k, m, t} L_{k, m, t} \geq r_{k, \tau}^{\mathrm{Req}}, \quad \tau \in 1,2, \ldots, T, \quad \tau<d_{i}^{\mathrm{RT}}(20)
$$

where, $r_{i, \tau}^{\text {Req }}$ is the required number of bits that needs to be transmitted in $\tau$ time slots.

We consider the minimum required power used for QoS support. There is the following OP:
OP-2:

$$
\min \sum_{t=1}^{\tau} \sum_{m=1}^{M} \sum_{k=1}^{K}\left(2^{\frac{R_{k, m, t}}{\Delta f}}-1\right) \frac{L_{k, m, t}}{\beta_{k, m, t}}
$$

s.t. Equations (16) and (17) satisfies

$$
\sum_{t=1}^{\tau} \sum_{m=1}^{M} R_{k, m, t} L_{k, m, t}=r_{k, \tau}^{\mathrm{Req}}, \quad \forall k \in \mathcal{K}
$$

The objective of OP-2 is to minimize the required power and satisfy the QoS requirements at the same time. For the solution of OP-2, there is the following proposition:

Propositon 2: As $M \rightarrow \infty$, for $\forall t \in \mathcal{T}$, the optimal solution $\left\{R_{k, m, t}^{*}, L_{k, m, t}^{*}\right\}$ for OP-2 satisfies

$$
\sum_{m=1}^{M} R_{k, m, t}^{*} L_{k, m, t}^{*}=r_{k, t}^{\mathrm{Req}}, \quad \forall t \in \mathcal{T}
$$

where, $r_{k, t}^{\mathrm{Req}}=\left\{\begin{array}{l}l_{i}^{\mathrm{RT}} / d_{i}^{\mathrm{RT}} \quad \forall i \in \mathcal{I} \\ R_{j}^{\mathrm{NRT}} / T_{s}, \forall j \in \mathcal{J}\end{array}\right.$, is the required bit rate at each time slot.

Proof. Details are provided in Appendix 2.

In Proposition 2, we assumed that $M \rightarrow \infty$, and Equation (23) shows that the required rate needed to be transmitted at each time slot is the same for any allocation time. However, in this paper, $M$ is finite, in order to utilize the Proposition 2 to simplify the OP-1, we evaluated the average minimum QoS-guaranteed power per time slot with different $\tau$, which has been shown in Figure 2.

In this simulation, we assume that all the SUs are with RT service, the subcarrier spacing is $16 \mathrm{KHz}$, and the required data rate is $R_{k}=800 \mathrm{kbps}$, so the packet length is $r_{k, \tau}^{\text {Req }}=R_{k} T_{s} \tau$. Let the $\mathrm{SU}$ number be 3 , and the value of $\tau$ is $1,3,5$, and 8, respectively. From Figure 2, we can see that for different value of $\tau$, the average QoS-guaranteed power is different. When the number of available subcarriers per time slot $M$ is increased, the effect of $\tau$ value over the QoS-guaranteed power is smaller. When $M=64$, the difference between the values of ordinate is less than 0.05 ; when $M$ increased to 128 , the difference is only 0.027 . It means that when $M$ becomes large, enough selectivity and diversity can be obtained for the system to achieve nearly the same minimum power for different $\tau$ values. Thus, based on Proposition 2, a reasonable approximation can be provided to transform the QoS constraints during $\tau$ time slots to one time slot with a reasonable number of subcarriers $M$ [18]. 


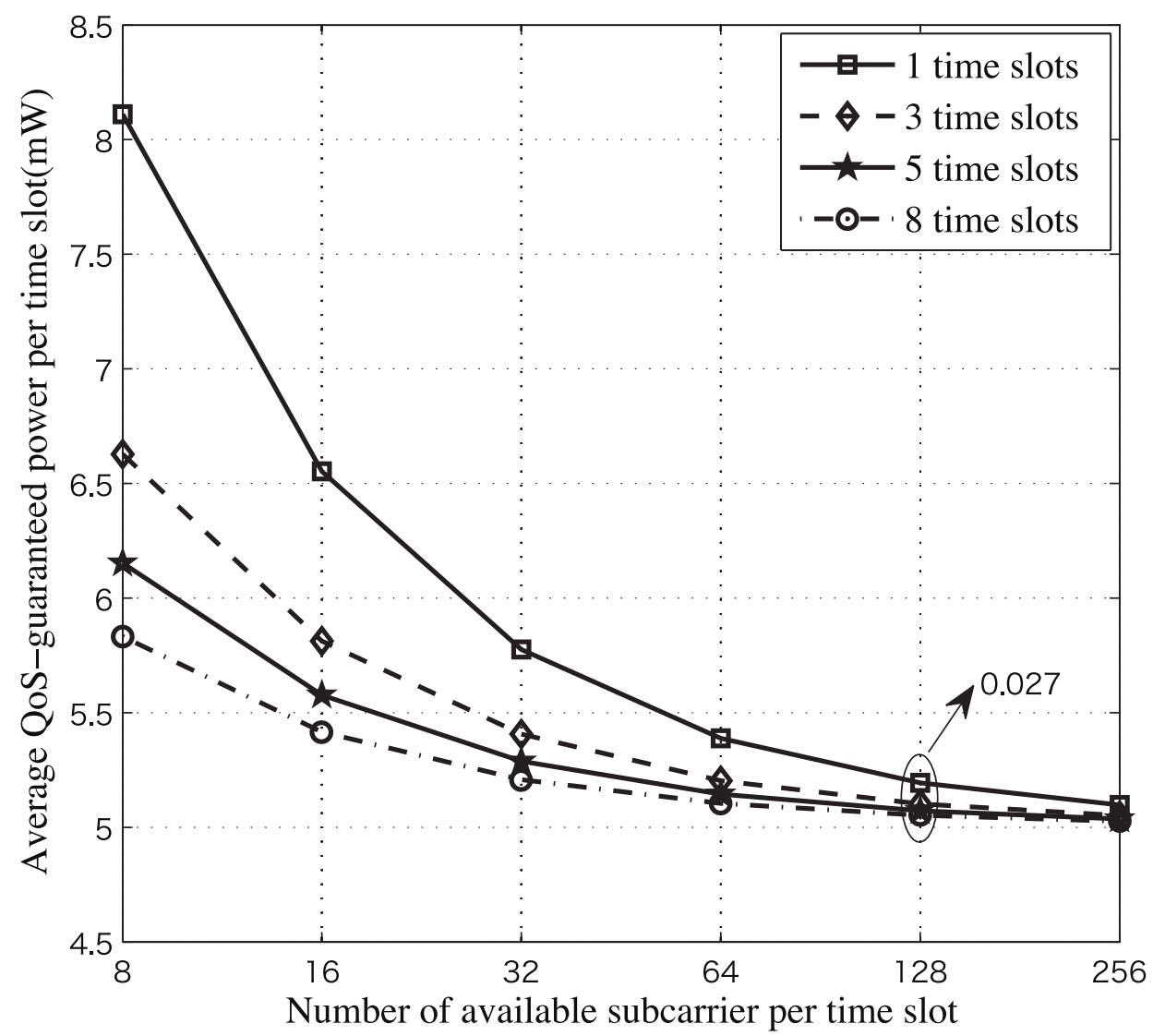

Figure 2 The minimum QoS-guaranteed power per time slot when $K=3, \tau=1,3,5,8$.

Therefore, the MAC-layer QoS constraints (11)-(13) in OP-1 can be replaced by the following PHY-layer constraint in one time slot:

$$
\sum_{m=1}^{M} R_{k, m, t} L_{k, m, t} \geq r_{k}^{\mathrm{Req}}
$$

Assume that there are enough subcarriers to satisfy the requirements of multiple services at each time slot. In order to derive the solutions, we introduced the surplus variable $\lambda_{k}$ [19] into the constraint (24), then, (24) is replaced by:

$$
\sum_{m=1}^{M} R_{k, m, t} L_{k, m, t}=r_{k}^{\mathrm{Req}}+\lambda_{k}, \quad \lambda_{k} \geq 0
$$

where, $\lambda_{k}$ is the surplus variable for SU $k$ and represents the amount by which the total allocated rate is exceeded. Moreover, the larger the value of $\lambda_{k}$, the higher the value of the system throughput; thus, $\lambda_{k}$ should be maximized in each time slot, then, the system utility function (14) in OP-1 can be replaced by the maximization of $\lambda_{k}$.

\subsection{QoS-guaranteed RA algorithm}

Due to the analysis and the simplifications above, we can describe the algorithm as following. Since the problem only depends on the parameters in the current time slot, the time index $t$ can be removed for simplicity. Therefore, the OP of joint cross-layer RA can be described as:

OP-3:

$$
\max \sum_{k=1}^{K} \lambda_{k}
$$

s.t.

$$
0 \leq P_{k, m} \leq P_{m}^{\max }, \sum_{k=1}^{K} \sum_{m=1}^{M} P_{k, m} \leq P_{0}
$$




$$
\begin{aligned}
& \sum_{k=1}^{K} L_{k, m} \leq 1, \quad L_{k, m}=\{0,1\} \\
& 0 \leq R_{k, m} \leq R_{\max } \\
& \sum_{m=1}^{M} R_{k, m} L_{k, m}=r_{k}^{\text {Req }}+\lambda_{k}, \quad \lambda_{k} \geq 0
\end{aligned}
$$

Optimization problem-3 is a constrained nonlinear programming problem, and in general, is intractable. It is shown in Appendix 3 that Problem OP-3 can be decomposed into three subproblems. The following optimal solution can be obatined using Lagrangian duality based technique $[19,20]$.

Let $R_{k, m^{\prime}}^{*} P_{k, m^{\prime}}^{*} L_{k, m}^{*}, k^{*}, \lambda_{k}^{*}, \xi_{k, m}^{*}, \varphi^{*}, \eta_{k}^{*}, \varsigma_{k}^{*}$ be an optimal solution set, where, $\xi_{k, m}, \varphi, \eta_{k}, \varsigma_{k}, k \in \mathcal{K}, m \in \mathcal{M}$ are the non-negative Lagrange multipliers [19], see details in Appendix C. Moreover, $R_{k, m}$ is replaced by $\widehat{R}_{k, m} \Delta f$, so, $\widehat{R}_{\max }=R_{\max } / \Delta f$ in the following equations.

Solution $S^{*}$ : The optimal solution $S^{*}=\left\{P_{k, m^{\prime}}^{*} L_{k, m^{\prime}}^{*} k^{*}\right\}$ for Problem OP-3 has the following properties.

(1) For a given $\mathrm{SU} k$, if the subcarrier allocation $L_{k, m}^{*}=1$, the optimal power allocation strategy is:

$$
P_{k, m}^{*}= \begin{cases}0, & \omega_{k}<\frac{\ln (2)}{\Delta f \beta_{k, m}} \\ \frac{\omega_{k} \Delta f}{\ln (2)}-\frac{1}{\beta_{k, m}}, & \frac{\ln (2)}{\Delta f \beta_{k, m}} \leq \omega_{k} \leq \frac{2^{\widehat{R}_{\max }} \ln (2)}{\Delta f \beta_{k, m}}(31) \\ \frac{2^{\widehat{R}_{\max }}-1}{\beta_{k, m}}, & \omega_{k}>\frac{2^{\widehat{R}_{\max }} \ln (2)}{\Delta f \beta_{k, m}}\end{cases}
$$

Where, $\omega_{k}=\frac{\eta_{k}^{*} L_{k, m}^{*}}{\xi_{k, m}^{*}+\varphi^{*}}$, represents the update of the multiplier. Moreover, $\omega_{k}$ can be viewed as the IWF level for SU $k$ and will be discussed later. From (31), the rate allocation $R_{k, m}^{*}$ and $\widehat{R}_{k, m}^{*}$ can be obtained by using (10).

(2) The subcarrier allocation strategy for subcarrier $m$ is:

$$
\begin{aligned}
& L_{k, m}^{*}=\left\{\begin{array}{l}
1, k=k^{*} \text { and } m \in \mathcal{M} \\
0, \text { otherwise }
\end{array}\right. \\
& k^{*}=\arg \max \quad \eta_{k}^{*} \widehat{R}_{k, m}^{*}
\end{aligned}
$$

Suppose that for a given $t$ and $m$, the values of $\eta_{k}^{*} \widehat{R}_{k, m}^{*}$ are the same for several users, we will choose one SU arbitrarily.
(3) IWF level $\omega_{k}$ is:

$$
\omega_{k}= \begin{cases}\omega_{B}, & \lambda_{k}^{*}>0 \\ \left(1+\varsigma_{k}^{*}\right) \omega_{B}, & \lambda_{k}^{*}=0\end{cases}
$$

where, $\omega_{B}=1 /\left(\xi_{k, m}^{*}+\varphi^{*}\right), \xi_{k, m}^{*}>0, \varphi^{*}>0$.

Proof. Details are provided in Appendix 3.

We can know from the above optimal values, if the water-filling levels $\left\{\omega_{k}\right\}$ for all SUs can be found, the optimal power and subcarrier allocation are obtained from (31) and (32).

In the system, we assume the primary bandwidth is large enough for SUs to guarantee the QoS, that is, according to Figure 2, $M>64$. There are two phases in our algorithm. The first one is to provide QoS support for all SUs. Then, it is the system throughput maximization. According to (31) and (10), the higher the value of water-filling level $\omega_{k}$, the higher the number of allocated bit rate for SU $k$. So, at the starting point, $\omega_{B}$ should be set to be the lowest water-filling level among all SUs. At each iteration, $\omega_{B}$ is increased to the next level that is higher than before. Based on the water-filling levels, the allocation algorithm is performed according to (31), (32), and (33). The process will stop when $\sum_{k=1}^{K} \sum_{m=1}^{M} P_{k, m}^{*} \geq P_{0}$ or the subcarriers have been used out. If $\sum_{k=1}^{K} \sum_{m=1}^{M} P_{k, m}^{*}>P_{0}$, the algorithm will use (31) to find an appropriate value of $\omega_{k}$ that satisfies the KKT condition (70), see Appendix 3.

A brief description of the procedure is given as follows:

(i) Initialize the water-filling level $\omega_{k}, \omega_{B}$.

(ii) In Phase I, for $m=1, \ldots, M$, do the following:

- For $k=1, \ldots, K$, do subcarrier and power allocation using (31), (32), and (33).

- Check if the QoS requirements have been satisfied. If for all SUs, $\sum_{m=1}^{M} R_{k, m}^{*} L_{k, m}^{*} \geq r_{k}^{\text {Req }}$, go to step (iii). If no, find the SUs whose bit rates less than $r_{k}^{\text {Req }}$ as set $K^{-}$, and find the SUs whose bit rates greater than $r_{k}^{\text {Req }}$ as set $K^{+}$. Adjust $\omega_{k}$ for SUs in set $K^{-}$and $K^{+}$, and reset $L_{k, m}^{*}=0, k \in K^{-} \cup K^{+}$, reallocate the left subcarriers and power to SUs in $K^{-}$and $K^{+}$, until $\sum_{m=1}^{M} R_{k, m}^{*} L_{k, m}^{*}=r_{k}^{\text {Req }}$ or $m=M$, then go to step (iii). (iii) Check if the transmit power and the power limits have been fulfilled. First, check if $P_{k, m}^{*} \leq P_{m}^{\max }$. If no, update $\omega_{k}$ until $P_{k, m}^{*} \leq P_{m}^{\max }$. Next, compare $\sum_{k=1}^{K} \sum_{m=1}^{M} P_{k, m}^{*}$ with $P_{0}$. If $\sum_{k=1}^{K} \sum_{m=1}^{M} P_{k, m}^{*}=P_{0}$, 
the algorithm will be finished. If $\sum_{k=1}^{K} \sum_{m=1}^{M} P_{k, m}^{*}<P_{0}$, go to step (iv). If $\sum_{k=1}^{K} \sum_{m=1}^{M} P_{k, m}^{*}>P_{0}$, adjust the base water-filling level $\omega_{B}$ to a smaller one, then back to step (ii).

(iv) In Phase II, the power has not been used out. We need to adjust $\omega_{B}$ to a higher value, and allocate all the resource to the SUs using (31), (32), and (33) to maximize the system throughput.

\section{Computer simulation}

In this section, simulations are performed for the downlink OFDMA-based MCR system to evaluate the effectiveness of the proposed algorithm. The simulation parameters are summarized in Table 1 . The simulation area is $2 \mathrm{~km} \times 2 \mathrm{~km}$ with the CBS located at the positions $d_{\mathrm{sp}}$ far away from the PBS, where $d_{\mathrm{sp}}$ is the distance between two BSs and varies from 0 to $1 \mathrm{~km}$. It is assumed that the channel gain is constant during $1 \mathrm{~ms}$ periods, thus RA is performed once every $1 \mathrm{~ms}$, which is also called one scheduling time. We assume the wireless propagation environment is urban area, and the cell radius is $500 \mathrm{~m}$. PUs and SUs are randomly located in its own cell area at each scheduling time. All performance results are obtained by over 1,000 simulation runs.

Moreover, we assume the PS is always downlink transmissions in the simulation. So, the impact of TDD frame ratio will not be considered here, since the performance with considering the frame ratio is proportional to the evaluate value, and not difficult to deduce.

Table 1 Simulation parameters

\begin{tabular}{ll}
\hline Parameters & Value \\
\hline Number of PUs $N$ & 8 \\
Number of SUs $M$ & 10 \\
System bandwidth $B_{w}$ & $2 \mathrm{MHz}$ \\
System center frequency $f_{c}$ & $1.9 \mathrm{GHz}$ \\
Total power at the PBS & $10 \mathrm{~W}$ \\
Total power at the CBS $P_{0}$ & $13 \mathrm{~W}$ \\
Predefined SINR of PUs in dB $\gamma_{p}^{\mathrm{dB}}$ & $10 \mathrm{~dB}$ \\
Interference violation probability $\sigma^{(p)}$ & 0.01 \\
Antenna gain $G_{s}$ & $8 \mathrm{dBi}$ \\
Number of subcarriers $M$ & 128 \\
Path loss exponent $\alpha$ & 4 \\
Number of RT SUs / & 5 \\
Number of NRT SUs $J$ & 5 \\
Delay of RT service $d_{i}^{\mathrm{RT}}$ (time slots) & 90 \\
Symbol period $T_{s}$ & $40 \mu \mathrm{bits}$ \\
Real-time data rate $R^{\text {RT }}$ (kbps) & $300 \mathrm{kbps}$ \\
Required average data rate $R_{j}^{\mathrm{NRT}}$ & \\
Max. number of bits per subcarrier $r_{\text {max }}$ & $500]$ \\
\hline
\end{tabular}

\subsection{Effectiveness of proposed design}

Firstly, we consider the worst case that all the primary bandwidth has been uniformly allocated to PUs, and each with $250 \mathrm{kHz}$ bandwidth, that means there is no non-active PU bands in the PS. The transmit power at the PBS is uniformly allocated to eight PUs. In this case, if the CR system is overlapped with PS, the interference power limits $I_{m}^{\mathrm{lim}}$ may be very small, even zero. In order to share the bandwidth with the PS, the distance between two BSs $d_{\text {sp }}$ should be large enough, then, the SU-to-PU interference can be controlled. Here, we set $d_{\mathrm{sp}}=100 \mathrm{~m}$ at first.

For comparison, we study two conventional RA schemes: channel greedy with power control and proportional fairness (PF) with equal power. For traditional OFDMA systems, channel greedy scheduling with water-filling/equal power allocation in [21], and PF scheduling with water-fill/equal power allocation in [22], have been proposed. However, OFDMA-based CR systems are different from traditional ones. Here, we would like to compare the proposed design to these two schemes to indicate that our proposed scheme is more suitable for CR systems. The first conventional scheme allocates the sub-carriers to the SU who has the largest SINR on the considered subcarrier, and allocates the power based on the power limits to control the interference. The second one assigns the subcarriers uniformly to all SUs for fairness, and equal power to all subcarriers.

Figures 3 and 4 are CDF of achieved SINR and relative interference power of PU 1, which is located at the cell edge of the PS, respectively. The relative interference power is defined as $10 \log _{10}\left(I_{n}^{\max } / I_{\text {siml }}\right)(\mathrm{dB})$, where, $I_{\text {siml }}$ is the simulated interference power. From Figures 3 and 4 , we can see that the PF scheme cannot provide the PUs with predefined SINR because of the high interference. Compared to Greedy scheme and PF scheme, the proposed method not only achieves the highest SINR, which is much higher than predefined value, but also controls the SU-to-PU interference well.

In Figures 5 and 6, the simulation results of QoS support for RT and NRT SUs are shown respectively. In Figure 5 , we set $d_{2}=500 \mathrm{~m}, d_{5}=100 \mathrm{~m}$, where, $d_{2}$ and $d_{5}$ are the distances from SU 2 and SU 5 to the CBS, respectively. We can see from this figure that $\mathrm{SU} 2$, which is the cell edge user with great channel fading, can only obtain the basic QoS-guaranteed data rate 600 kbps; however, SU 5, which is near to the CBS with good channel state, can achieve much higher rate so as to maximize the system throughput. Figure 6 shows the average data rate of NRT SUs versus different RT data rate. Compared to conventinal schemes, the proposed design can achieve much higher average data rate. 


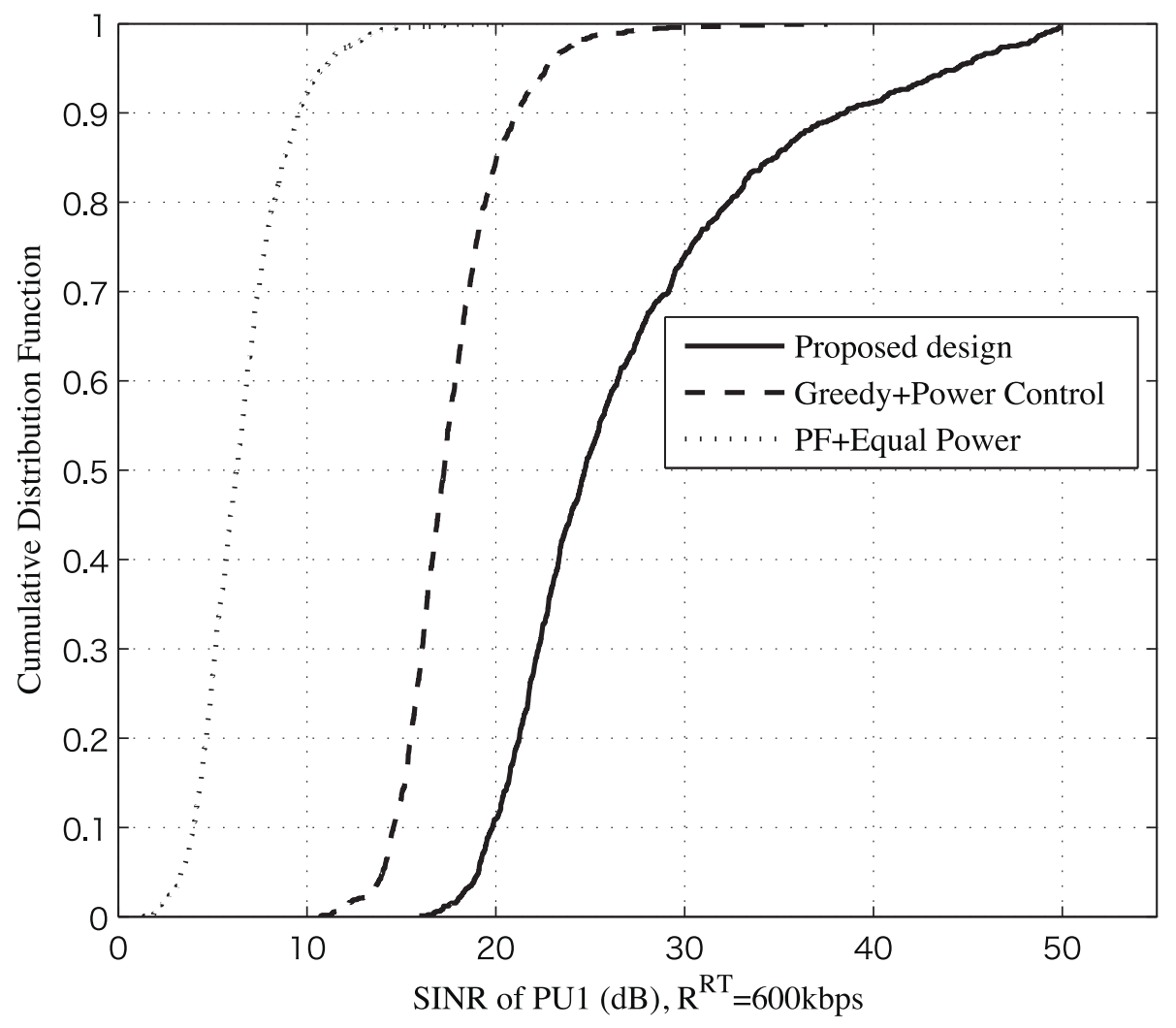

Figure 3 CDF of SINR of PU 1

Figure 7 shows the sum rate of the CR system. Compared to the other two RA schemes, it yields a significant higher sum rate. For different $\mathrm{RT}$ data rate, the sum rate of $C R$ system is almost the same due to the same available CBS power and spectrum resource. For the Greedy scheme, even though it can control the SUto-PU interference, the spectrum efficiency is really low. From Figure 7, we can see the proposed design has the best performance.

\subsection{Performance comparison between spectrum underlay and overlay}

In practical systems, two systems may be overlapped with each other, and the primary bandwidth may not be used out, that is, the probability of non-active PU bands satisfies $P_{\text {non-active }} \geq 0$.

When CBS and PBS are too close to each other and the primary bands are all in use, it is difficult to control the MI with power control, or to provide satisfactory QoS to all SUs. Figure 8 is the CDF of $\lambda_{k}$ at different CBS-PBS distance $d_{\mathrm{sp}}$, which has been changed from 350 to $650 \mathrm{~m}$. Here, the simulation parameters are the same with Section 5.1 and $P_{\text {non-active }}=0$. We define $\operatorname{Pr}$ $\left\{\lambda_{k}<0\right\}=\delta^{(s)}$, which is the secondary QoS-unsatisfactory probability. When $\delta^{(s)}>0.01$, we consider that the CR system cannot guarantee the QoS for all SUs. From Figure 8, we can see that in order to limit the interference at the PUs and provide the QoS for ten SUs as well, $d_{\mathrm{sp}}$ cannot be very small. When $d_{\mathrm{sp}}=650 \mathrm{~m}, \delta^{(s)}$ is only 0.005 ; while $d_{\mathrm{sp}}$ decreased to $550 \mathrm{~m}, \delta^{(s)}$ increased to 0.029 . Then, we consider that the CR system when $d_{\mathrm{sp}}=550 \mathrm{~m}$ cannot satisfy the requirements for all SUs at the same time. In this situation, we should choose other better sharing methods for the CR system.

Therefore, in order to improve the effectiveness of the coexistent system, we compared the performance between spectrum underlay and spectrum overlay sharing methods.

Figures 9 and 10 are the average maximum number of SUs and the sum rate of CR system, respectively. In these two figures, we consider two different sharing schemes: spectrum overlay (only available non-active subbands can be utilized), and spectrum underlay \& overlay (the whole primary bands can be utilized, and 


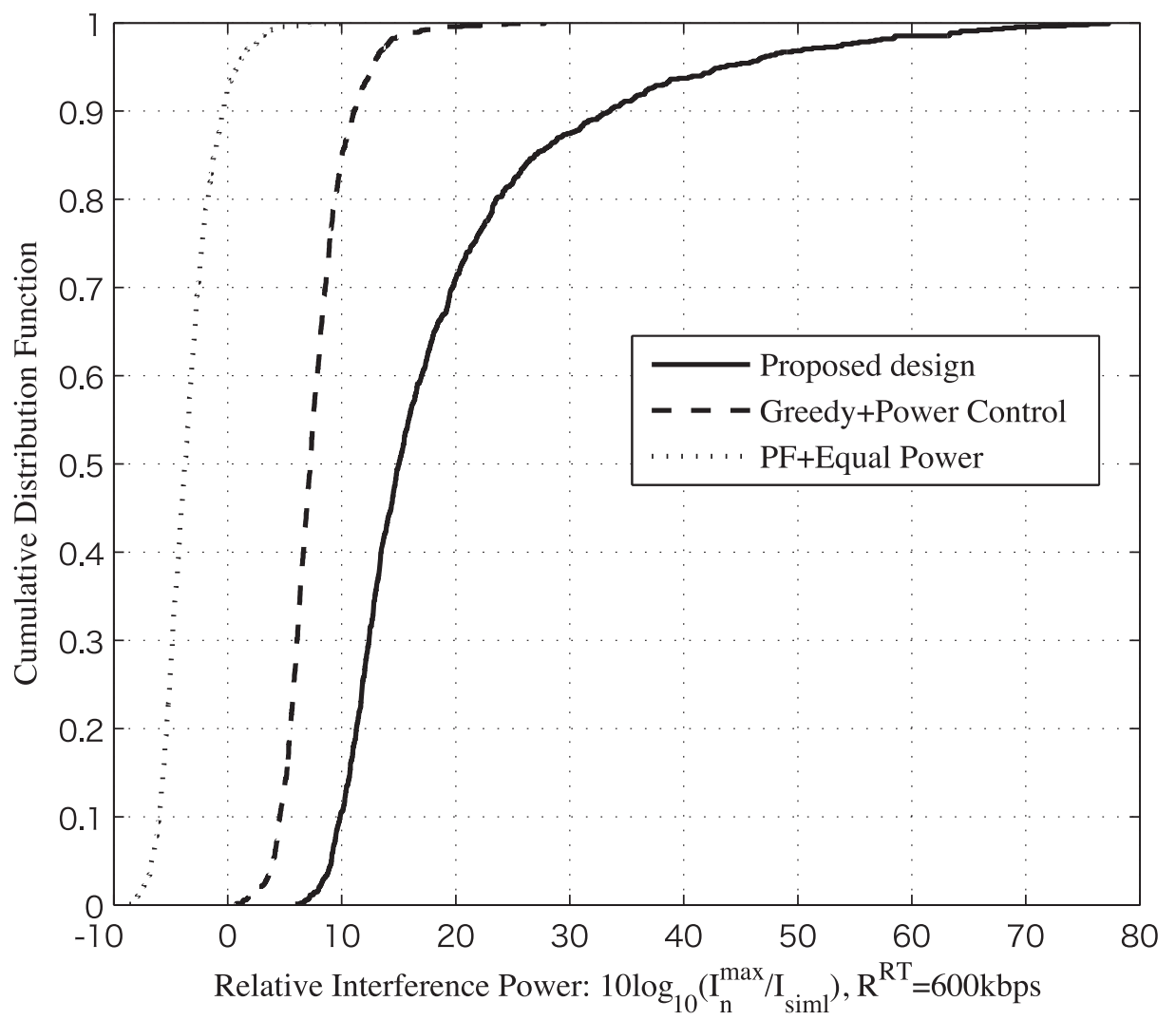

Figure 4 CDF of relative interference of PU 1 .

control the interference level to the active subbands). We set the probability of non-active PU bands to be $P_{\text {non-active }}=0$ or $20 \%$. When $P_{\text {non-active }}=0$, only the spectrum underlay sharing can be utilized for the CR system; while, when $P_{\text {non-active }}=20 \%$, both the spectrum underlay and spectrum overlay are available for the CR system to share the spectrum with PS.

From Figures 9 and 10, we can see that the maximum RT/NRT SU number and the sum rate of the CR system are increasing with the distance between the CBS and the PBS when utilizing the spectrum underlay scheme for both $P_{\text {non-active }}=0$ and $20 \%$. However, the performance of the spectrum overlay sharing scheme is almost the same, and only related to $P_{\text {non-active }}=0$, which is the available spectrum resource. Compared the performance of these sharing schemes in Figures 9 and 10, the spectrum underlay has substantial higher spectrum efficiency and can be utilized for both $P_{\text {non-active }}=0$ and $20 \%$. When $P_{\text {non-active }}=0$, the spectrum underlay \& overlay sharing method is the best candidate for CR systems to access unlicensed spectrum.

\section{Conclusions}

A combined cross-layer RA and interference avoidance optimization design for downlink OFDMA-based MCR systems has been proposed. We utilize a predefined SINR and an interference violation probability at the primary receivers for the power allocation and interference control. QoS constraints transformation and IWF method have been analyzed to maximize the system throughput for the CR system and provide satisfactory QoS support for different services of the SUs. To obtain optimal solution, we developed a convex OP to solve the system utility function.

Compared to the conventional RA schemes, the proposed cross-layer design with the spectrum underlay sharing method could share the spectrum with the PUs more effectively. Simulation results illustrate that our proposed design has a significant performance gain. On the other hand, the comparision between the spectrum underlay and overlay has shown that if there is available subbands in the PS, it has the best spectrum efficiency with both the spectrum underlay and overlay sharing methods. 


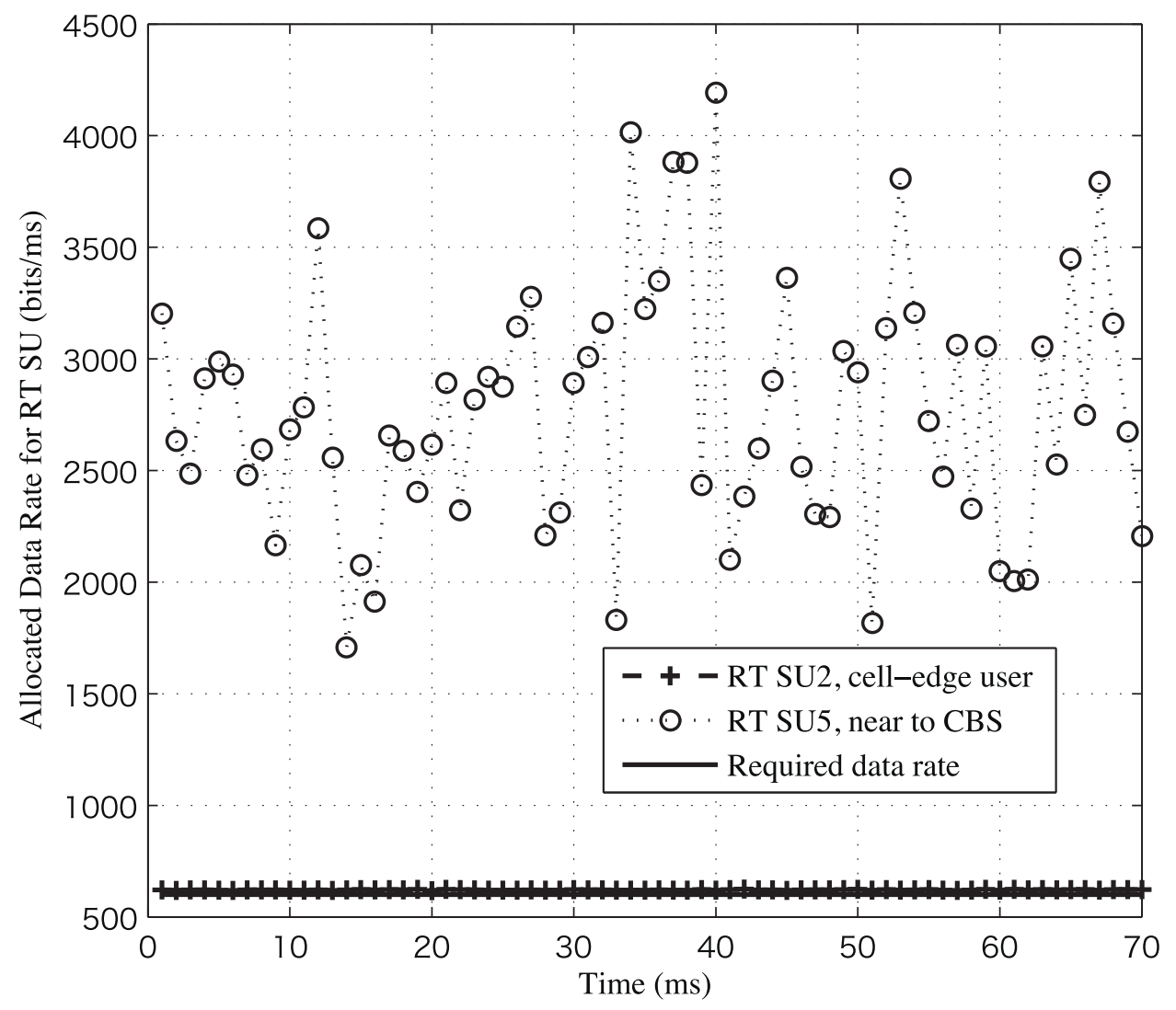

Figure 5 QoS support for RT SUs.

\section{Acknowledgements}

A part of this research has been supported by the Japanese Ministry of Internal Affairs and Communications under Strategic Information and Communications R\&D Promotion Programme (SCOPE).

\section{Appendix}

\section{Proof of proposition}

In (7), the CDF of $g_{n, m, t}^{\mathrm{sp}}$ is given by $F(x)=\operatorname{Pr}\{X \leq x\}=1-e^{\left(-x^{2} / 2 \sigma^{2}\right)}, x \in[0, \infty), \sigma>0$. Then, for Equation (7), there is the following derivation:

$$
\begin{aligned}
& \operatorname{Pr}\left\{P_{k, m, t} g_{n, m, t}^{\mathrm{sp}} \cdot \mu\left(d_{n, t}^{\mathrm{sp}} / d_{0}\right)^{-\alpha}>I_{m}^{\mathrm{lim}}\right\}=\operatorname{Pr}\left\{g_{n, m, t}^{\mathrm{sp}}>\frac{I_{m}^{\mathrm{im}}}{P_{k, m, t} \mu\left(d_{n, t}^{\mathrm{sp}} / d_{0}\right)^{-\alpha}}\right\} \\
& =1-\operatorname{Pr}\left\{g_{n, m, t}^{\mathrm{sp}} \leq \frac{I_{m}^{\mathrm{lim}}}{P_{k, m, t} \mu\left(d_{n, t}^{\mathrm{sp}} / d_{0}\right)^{-\alpha}}\right\}=1-F\left(\frac{I_{m}^{\mathrm{lim}}}{P_{k, m, t} \mu\left(d_{n, t}^{\mathrm{sp}} / d_{0}\right)^{-\alpha}}\right) \leq \delta^{(p)}
\end{aligned}
$$

Then, from (35), we has the following inequality:

$$
F\left(\frac{I_{m}^{\mathrm{lim}}}{P_{k, m, t} \mu\left(d_{n, t}^{\mathrm{sp}} / d_{0}\right)^{-\alpha}}\right) \geq 1-\delta^{(p)}
$$

Due to the monotone non-decreasing property of CDFs, from (36), the following power constraint on subcarrier $m$ can be obtained:

$$
\frac{I_{m}^{\mathrm{lim}}}{P_{k, m, t} \mu\left(d_{n, t}^{\mathrm{sp}} / d_{0}\right)^{-\alpha}} \geq F^{-1}\left(1-\delta^{(p)}\right)
$$

From (37), the power limits for secondary subcarriers can be derived based on the primary performance targets and the channel characteristics.

$$
P_{k . m, t} \leq \frac{I_{m}^{\mathrm{lim}}}{\mu\left(d_{n, t}^{\mathrm{sp}} / d_{0}\right)^{-\alpha} F^{-1}(1-\delta(p)}
$$

where, $P_{k, m, t}$ can be written as $P_{m, t}$ instead.

\section{Proof of proposition}

From OP-2, we have the following OP: 


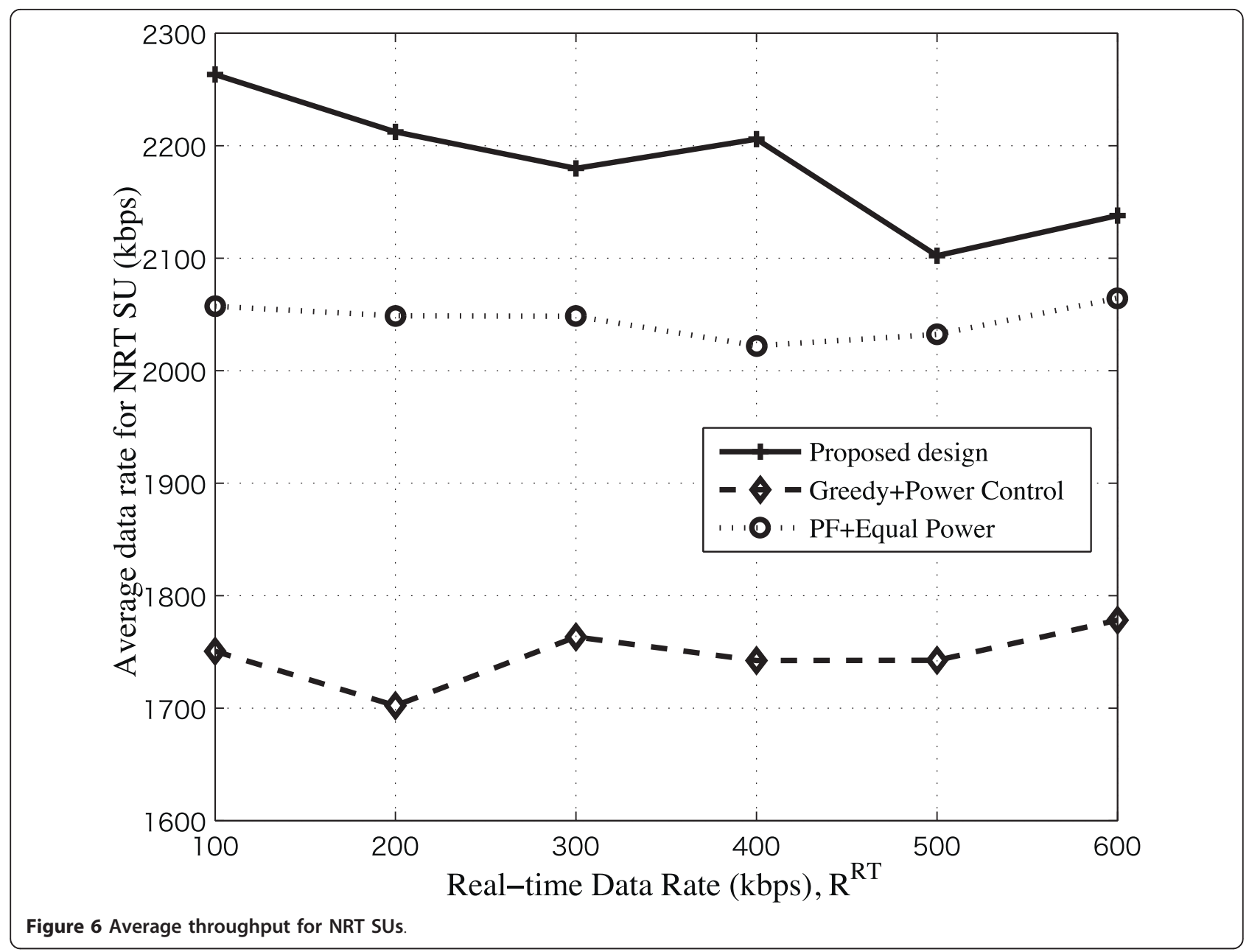

OP-A:

$$
\min \sum_{t=1}^{\tau} \sum_{m=1}^{M} \sum_{k=1}^{K}\left(2^{\frac{R_{\mathrm{k}, \mathrm{m}, \mathrm{t}}}{\Delta f}}-1\right) \frac{L_{k, m, t}}{\beta_{k, m, t}}
$$

s.t.

$$
\begin{aligned}
& \sum_{k=1}^{K} L_{k, m, t}-1 \leq 0, \quad L_{k, m, t}-1 \leq 0, \quad-L_{k, m, t} \leq 0 \\
& R_{k, m, t}-R_{\max } \leq 0, \quad-R_{k, m, t} \leq 0 \\
& r_{k, \tau}^{\text {Req }}-\sum_{t=1}^{\tau} \sum_{m=1}^{M} R_{k, m, t} L_{k, m, t}=0
\end{aligned}
$$

The functions (39) in OP-A are convex functions in convex set $\mathcal{C}=\left\{L_{k, m, t}=\{0,1\}, R_{k, m, t} \in\left[0, R_{\max }\right]\right\}$. The largrangian function [19] of the above convex optimization OP-A is:

$$
\begin{aligned}
& L=\sum_{t=1}^{\tau} \sum_{m=1}^{M} \sum_{k=1}^{K} \frac{\left(2^{\frac{R_{k, m, t}}{\Delta f}}-1\right) L_{k, m, t}}{\beta_{k, m, t}}+\sum_{t=1}^{\tau} \sum_{m=1}^{M} a_{m, t}\left(\sum_{k=1}^{K} L_{k, m, t}-1\right)+\sum_{t=1}^{\tau} \sum_{m=1}^{M} \sum_{k=1}^{K} b_{k, m, t}\left(L_{k, m, t}-1\right) \\
& -\sum_{t=1}^{T} \sum_{m=1}^{T} \sum_{k=1}^{K} c_{k, m, t} L_{k, m, t}+\sum_{t=1}^{T} \sum_{m=1}^{M} \sum_{k=1}^{K} e_{k, m, t}\left(R_{k, m, t}-R_{\max }\right)-\sum_{t=1}^{T} \sum_{m=1}^{M} \sum_{k=1}^{K} f_{k m, t} R_{k, m, t} \\
& +\sum_{k=1}^{K} h_{k}\left(r_{k, t}^{\text {Req }}-\sum_{t=1}^{T} \sum_{m=1}^{M} R_{k, m, L_{i}} L_{k, m, t}\right)
\end{aligned}
$$

where $\left\{a_{m, t}, b_{k, m, t}, c_{k, m, t}, e_{k, m, t}, f_{k, m, t}, h_{k}\right\}, t=1,2, \ldots, \tau$, $k \in \mathcal{K}, m \in \mathcal{M}$ are the Lagrange multipliers, and each multiplier should be no less than zero.

Let $\left\{R_{k, m, t}^{*}, L_{k, m, t}^{*}, a_{m, t}^{*}, b_{k, m, t}^{*}, c_{k, m, t}^{*}, e_{k, m, t}^{*} f_{k, m, t}^{*}, h_{k}^{*}\right\}$ be an optimal solution. Then the Karush-Kuhn-Tucker (KKT) conditions are as following:

$$
\begin{aligned}
& a_{m, t}^{*} \geq 0, b_{k, m, t}^{*} \geq 0, c_{k, m, t}^{*} \geq 0, e_{k, m, t}^{*} \geq 0, f_{k, m, t}^{*} \geq 0, h_{k}^{*} \geq 0 \\
& a_{m, t}^{*}\left(\sum_{k=1}^{K} L_{k, m, t}^{*}-1\right)=0
\end{aligned}
$$




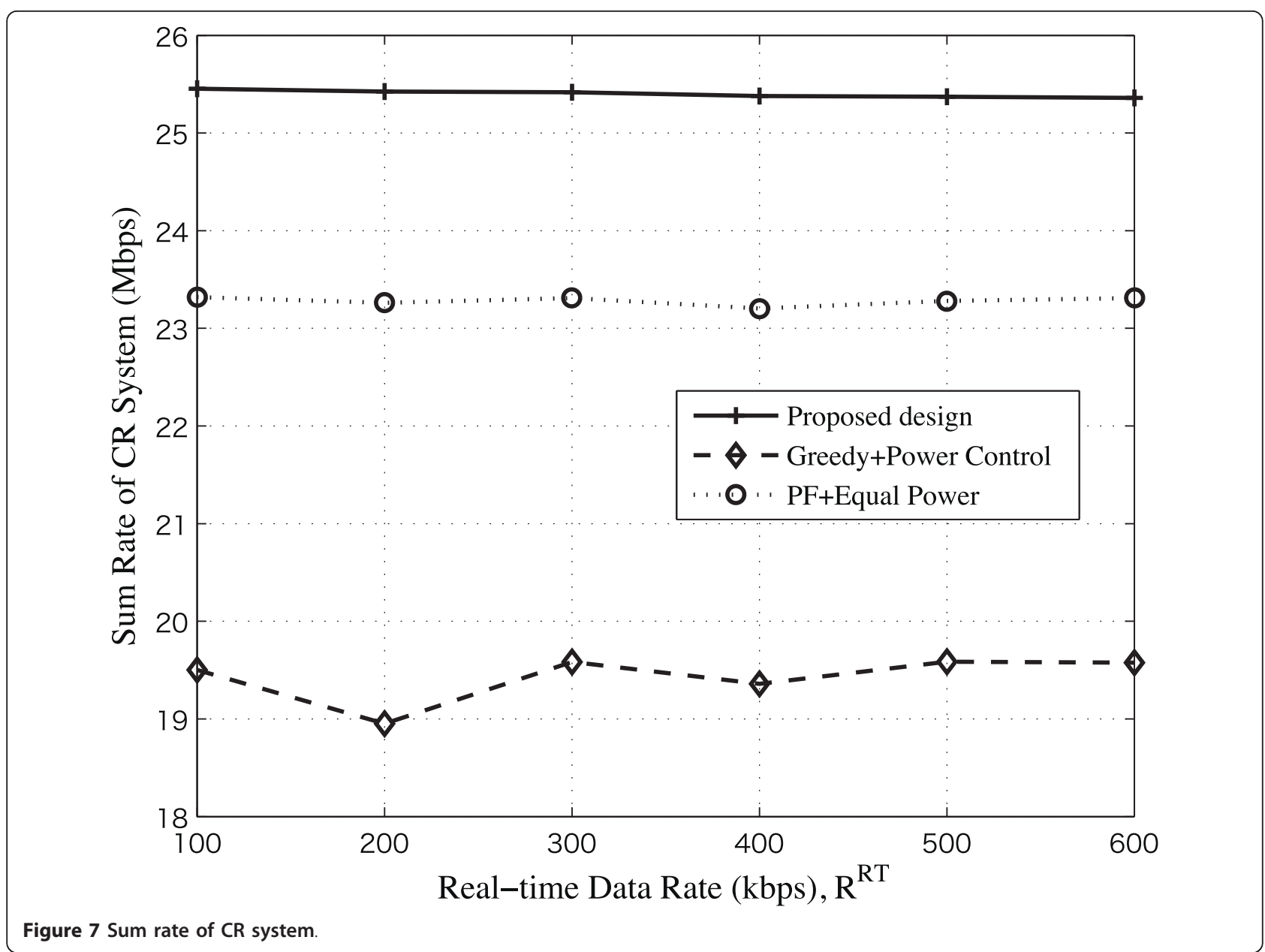

$$
\begin{aligned}
& b_{k, m, t}^{*}\left(L_{k, m, t}^{*}-1\right)=0 \\
& c_{k, m, t}^{*} L_{k, m, t}^{*}=0 \\
& e_{k, m, t}^{*}\left(R_{k, m, t}^{*}-R_{\max }\right)=0 \\
& f_{k, m, t}^{*} R_{k, m, t}^{*}=0 \\
& h_{k}^{*}\left(r_{k, \tau}^{\text {Req }}-\sum_{t=1}^{\tau} \sum_{m=1}^{M} R_{k, m, t}^{*} L_{k, m, t}^{*}\right)=0 \\
& \frac{L_{k, m, t}^{*} \ln (2) 2}{\beta_{k, m, t} \Delta f}+e_{k, m, t}^{*}-f_{k, m, t}^{*}-h_{k}^{*} L_{k, m, t}^{*}=0
\end{aligned}
$$

$$
\frac{\left(2^{\frac{R_{k, m, t}^{*}}{\Delta f}}-1\right)}{\beta_{k, m, t}}+a_{m, t}^{*}+b_{k, m, t}^{*}-c_{k, m, t}^{*}+h_{k}^{*} R_{k, m, t}^{*}=0
$$

Equations (51) and (52) are obtained by setting $\partial L /$ $\partial R_{k, m, t}=0$ and $\partial L / \partial L_{k, m, t}=0$, respectively.

From (51), when $L_{k, m, t}^{*} \neq 0$, we have

$$
R_{k, m, t}^{*}=\Delta f \log _{2}\left\{\frac{\left(h_{k}^{*} L_{k, m, t}^{*}+f_{k, m, t}^{*}-e_{k, m, t}^{*}\right) \beta_{k, m, t} \Delta f}{\ln (2) L_{k, m, t}^{*}}\right\}
$$

According to (48) and (49), $e_{k, m, t}^{*}$ and $f_{k, m, t}^{*}$ cannot be positive at the same time. Therefore, when $R_{k, m, t}^{*}=0, e_{k, m, t}^{*}=0, f_{k, m, t}^{*} \geq 0$, then, according to (53), $h_{k}^{*} \leq \frac{\ln (2)}{\beta_{k, m, t} \Delta f} ; \quad$ when $\quad R_{k, m, t}^{*}=R_{\max } e_{k, m, t}^{*} \geq 0, f_{k, m, t}^{*}=0$, 


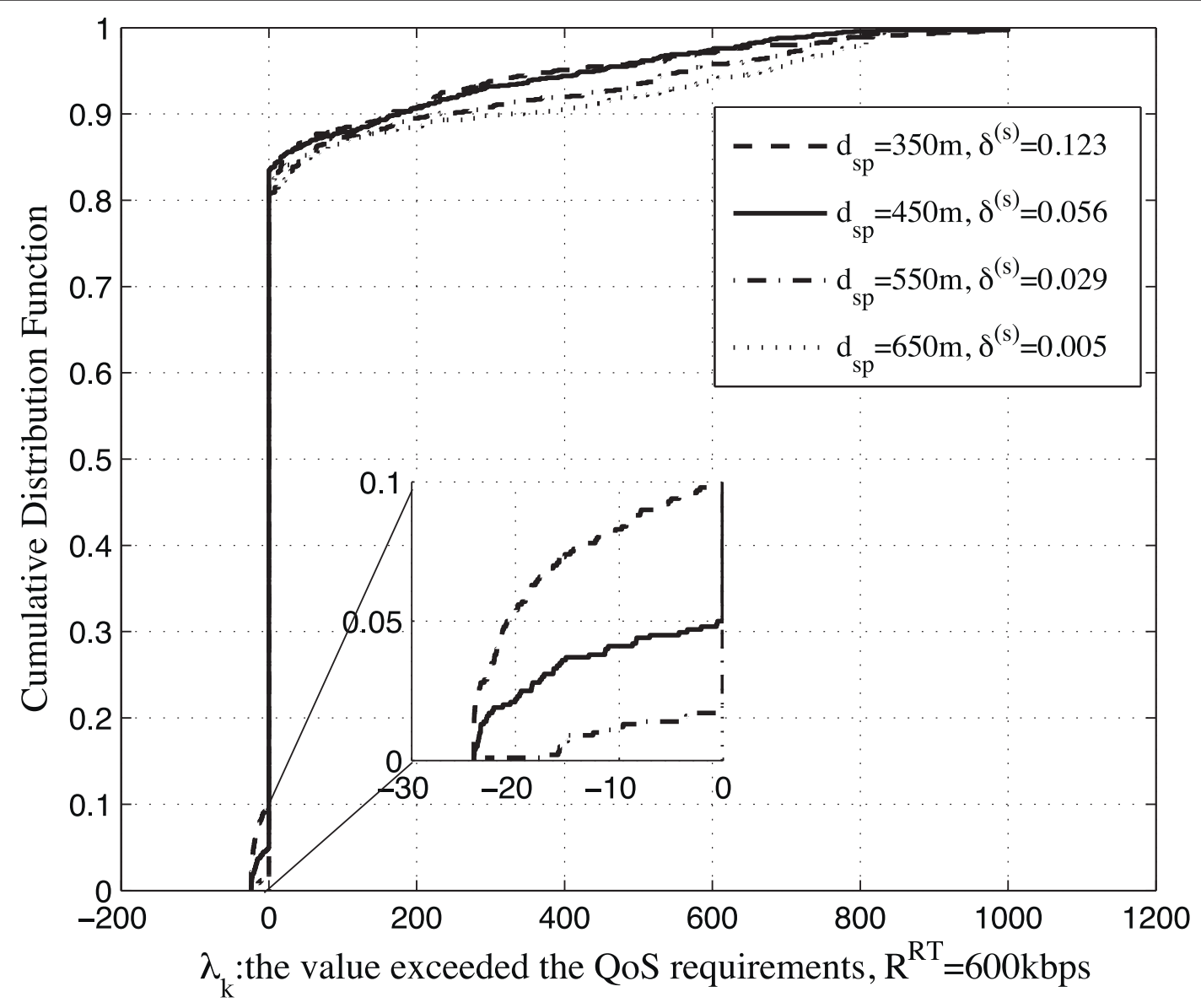

Figure $8 \mathrm{CDF}$ of the value exceeded the QoS requirements $\lambda_{k}$.

then, according to (53), $h_{k}^{*} \geq \frac{2^{\frac{R_{\max }}{\Delta f}} \ln (2)}{\beta_{k, m, t} \Delta f}$; when $0<R_{k, m, t}^{*}<R_{\max }, e_{k, m, t}^{*}=0, f_{k, m, t}^{*}=0$, then, according to (53), $\frac{\ln (2)}{\beta_{k, m, t} \Delta f}<h_{k}^{*}<\frac{2^{\frac{R_{\max }}{\Delta f}} \ln (2)}{\beta_{k, m, t} \Delta f}$. To summarize, the optimal rate allocation $R_{k, m, t}^{*}$ at time slot $t$ can be:

$$
R_{k, m, t}^{*}= \begin{cases}0, & h_{k}^{*} \leq \frac{\ln (2)}{\beta_{k, m, t} \Delta f} \\ \Delta f \log _{2}\left(\frac{h_{k}^{*} \beta_{k, m, t} \Delta f}{\ln (2)}\right), \frac{\ln (2)}{\beta_{k, m, t} \Delta f}<h_{k}^{*}<\frac{2^{\frac{R_{\max }}{\Delta f}} \ln (2)}{\beta_{k, m, t} \Delta f} \\ R_{\max ,} \\ h_{k}^{*} \geq \frac{2 \frac{R_{\max }^{\Delta f}}{\beta_{k, m, t} \Delta f} \ln (2)}{}\end{cases}
$$

Suppose that subcarrier $m$ has been allocated to more than one SU, that is, there exists $0<L_{k, m, t}^{*}<1$ for SUs $k_{1}, k_{2}, \ldots, k_{B}, B>1$. From (46) and (47), we have $b_{k, m, t}^{*}=0$ and $c_{k, m, t}^{*}=0$. Then, from (52), we have

$$
\frac{\left(2^{\frac{R_{k, m, t}^{*}}{\Delta f-1}}\right)}{\beta_{k, m, t}}+h_{k}^{*} R_{k, m, t}^{*}=-a_{m, t}^{*}
$$

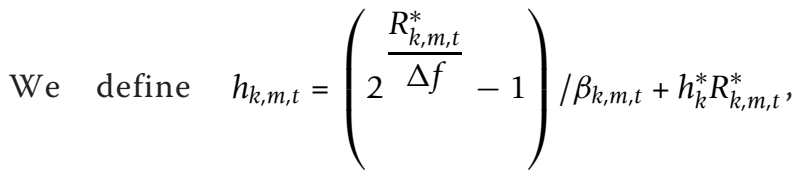
therefore, we have $h_{k, m, t}=-a_{m, t}^{*}$ for all $k=k_{1}, k_{2}, \ldots, k_{B}$, that is $h_{k_{1}, m, t}=h_{k_{2}, m, t}=\cdots=h_{k_{B}, m, t}$.

However, for the left side in (55), unless $\beta_{k, m, t}$ is equal for SUs $k_{1}, k_{2}, \ldots, k_{B}$, it is highly impossible that any of the two $h_{k, m, t}$ values will be equal. Since $\beta_{k, m, t}$ are channel state information, modeled as independent and random variables. Therefore, we conclude that for any time slot $t$ and subcarrier $m$, there is only one SU $k^{*}$, that is $L_{k^{*}, m, t}^{*}=1$ if subcarrier $m$ has been allocated. The 


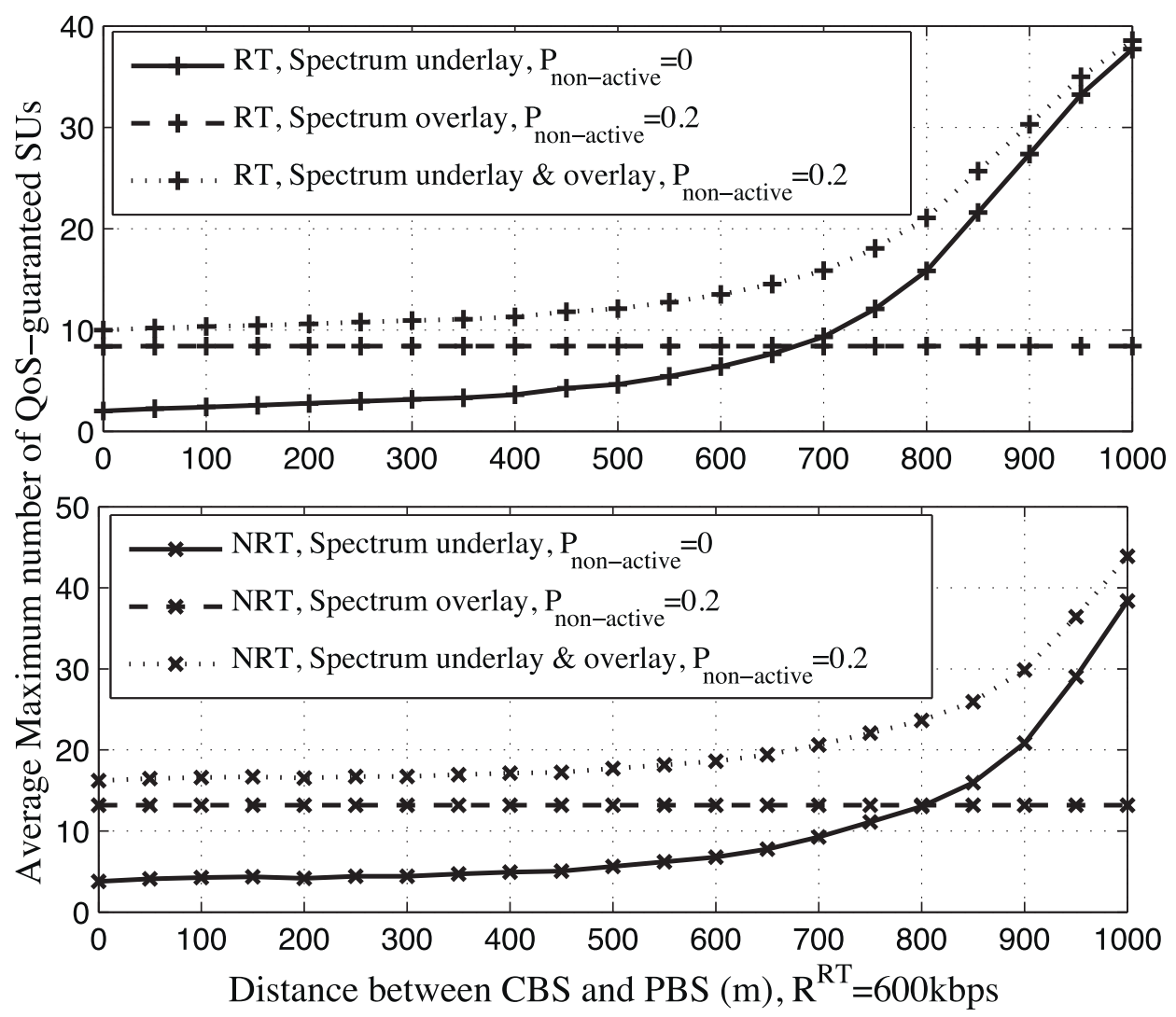

Figure 9 Maximum number of SUs with different sharing schemes.

method how to find this $\mathrm{SU}$, which is similar to that analyzed in Appendix 3, will be omitted here.

Now, the proof of Proposition 2 will continue. We consider $L_{k, m, t}^{*}=1$ for SU $k$. The allocated rates for $\mathrm{SU}$ $k$ during $\tau$ time slots $r_{k}$ can be calculated as following by using (54):

$$
r_{k}=\sum_{t=1}^{\tau} \sum_{m=1}^{M} R_{k, m, t}^{*}
$$

We define some probabilities:

$\operatorname{Pr}\{$ a given subcarrier is allocated to $\mathrm{SU} \mathrm{k}\}=p_{k}$;

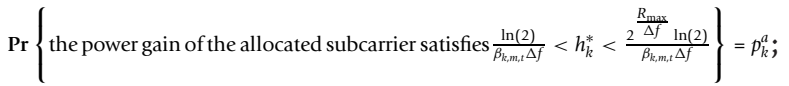

$\operatorname{Pr}\left\{\right.$ the power gain of the allocated subcarrier satisfies $\left.h_{k}^{*} \geq \frac{\frac{R_{m m}}{\Delta \Delta} \ln (2)}{\beta_{k m, t} \Delta f}\right\}=p_{k}^{b}$.

Equation (56) can be calculated as:

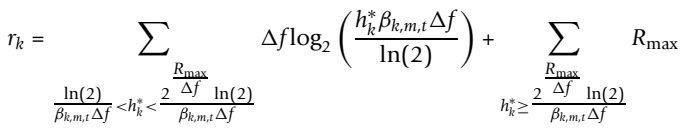

$$
\begin{aligned}
& =\sum_{t=1}^{\tau} m(t) p_{k} p_{k}^{a} \Delta f \log _{2}\left(\frac{h_{k}^{*} \beta_{k, m, t} \Delta f}{\ln (2)}\right)+\sum_{t=1}^{\tau} m(t) p_{k} b_{k}^{b} R_{\max }
\end{aligned}
$$

where, $m(t)$ is the available subcarrier number at time slot $t$.

Similarly, at time slot $t$, the optimal number of bit rate allocated to SU $k$ is obtained:

$$
\sum_{m=1}^{M} R_{k, m, t}^{*} L_{k, m, t}^{*}=m(t) p_{k}\left\{p_{k}^{a} \Delta f \log _{2}\left(\frac{h_{k}^{*} \beta_{k, m, t} \Delta f}{\ln (2)}\right)+p_{k}^{b} R_{\max }\right\}
$$

From (57) and (58), we have

$$
\sum_{m=1}^{M} R_{k, m, t}^{*} L_{k, m, t}^{*}=\frac{m(t)}{\sum_{t=1}^{\tau} m(t)} r_{k}
$$

Suppose that the allocation can achieve the QoS requirements for $\mathrm{SU} k$, therefore, the allocated bit rates during $\tau$ time slots satisfied $r_{k}=r_{k, t}^{\mathrm{Req}} \tau$, where, $r_{k, t}^{\mathrm{Req}}$ is the required bit rate at each time slot. So, Equation (59) can be rewritten as:

$$
\sum_{m=1}^{M} R_{k, m, t}^{*} L_{k, m, t}^{*}=\frac{m(t) \tau}{\sum_{t=1}^{\tau} m(t)} r_{k, t}^{\mathrm{Req}}
$$

Due to primary interference limits, not all the subcarriers are available for SUs. If the available bandwidth $m(t)$ is considerably small, only few bit rates can be allocated to 


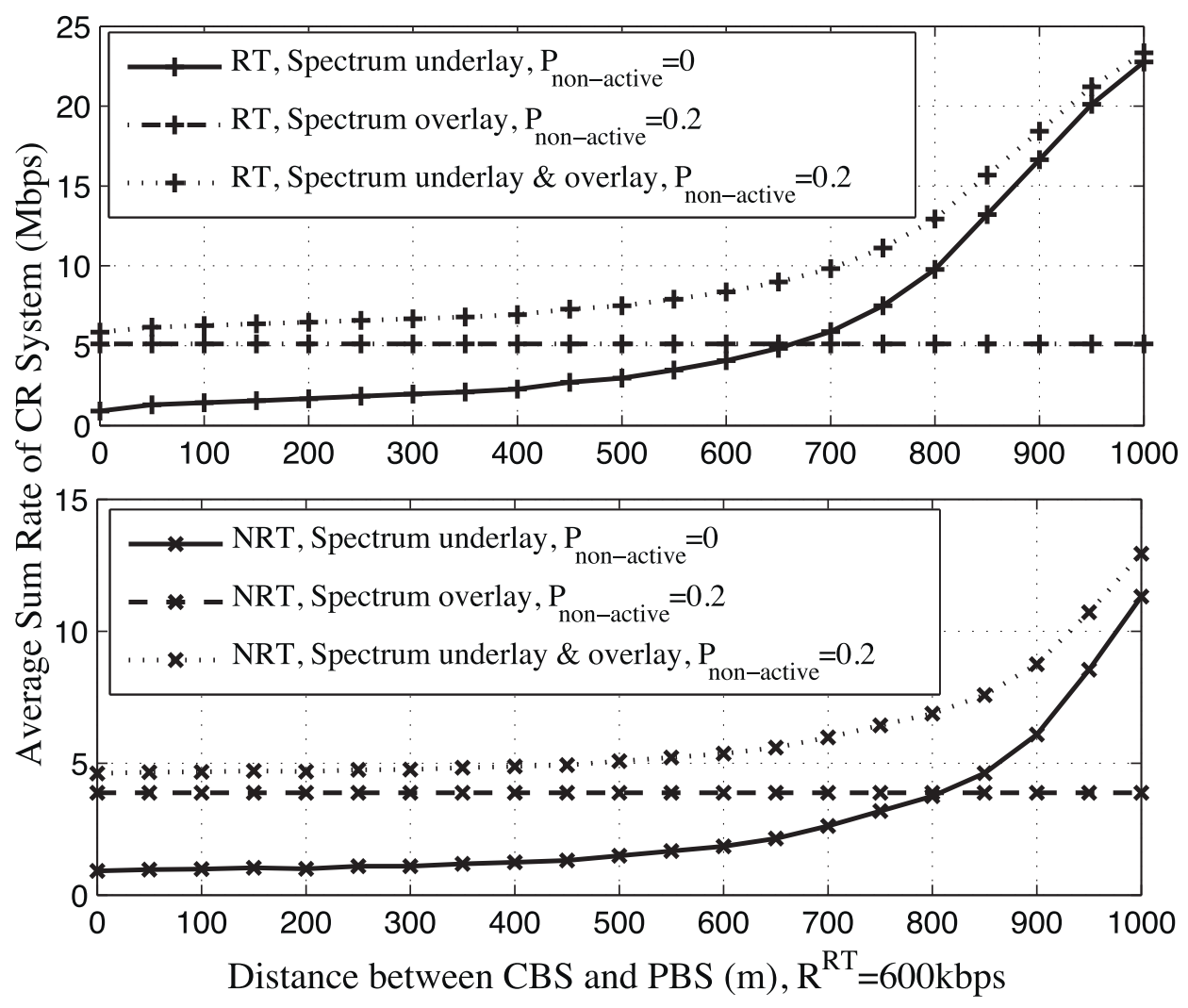

Figure 10 Sum rate of CR system with different sharing schemes

SU $k$ at time slot $t$. Then, it is difficult to satisfy the required bit rate $r_{k, t}^{\text {Req }}$ at each time slot, and the required bit rate during $\tau$ time slots also cannot be satisfied, since $r_{k}<r_{k, t}^{\text {Req }} \tau$ due to limited primary bandwidth. To guarantee the QoS requirments, $M$ needs to be large enough. Assume $M \rightarrow \infty$, so that at each time slot, there are $m(t) \geq$ $m_{\text {req }}$ that can achieve SUs' requirements, where, $m_{\text {req }}$ is the minimum number of required subcarrier. Therefore, for OP-A, to achieve the QoS and minimize the transmit power, $m(t)$ should be equal to $m_{\text {req }}$ at each time slot. If $M$ $\rightarrow \infty, \sum_{t=1}^{\tau} m(t)=\tau m_{\text {req }}$, and we can have:

$$
\sum_{m=1}^{M} R_{k, m, t}^{*} L_{k, m, t}^{*}=r_{k, t}^{\mathrm{Req}}
$$

\section{Proof of solution $S^{*}$}

In Problem OP-3, if we replace $R_{k, m}$ by $R_{k, m}=\widehat{R}_{k, m} \Delta f$, which can be obtained from (10). The following duality OP is obtained:
OP-B:

$$
\min \sum_{k=1}^{K}-\lambda_{k}
$$

s.t.

$$
P_{k, m}-P_{m}^{\max } \leq 0, \sum_{k=1}^{K} \sum_{m=1}^{M} P_{k, m}-P_{0} \leq 0
$$

$\sum_{k=1}^{K} L_{k, m}-1 \leq 0, \quad L_{k, m}-1 \leq 0, \quad-L_{k, m} \leq 0$

$\widehat{R}_{k, m} \Delta f-R_{\max } \leq 0, \quad-\widehat{R}_{k, m} \Delta f \leq 0$

$$
r_{k}^{\mathrm{Req}}+\lambda_{k}-\sum_{m=1}^{M} \widehat{R}_{k, m} \Delta f L_{k, m}=0, \quad-\lambda_{k} \leq 0
$$

where, $P_{k, m}=\frac{2^{\widehat{\widehat{R}}_{k, m}}-1}{\beta_{k, m}}$. 
The Lagrangian function $[19,20]$ associated with the above duality problem OP-B can be written as:

$$
\begin{aligned}
& L\left(\widehat{R}_{k, m}, L_{k, m}, \lambda_{k,}, \xi_{k, m}, \varphi, \psi_{m,}, \phi_{k, m}, v_{k, m}, \zeta_{k, m}, \varepsilon_{k, m}, \eta_{k,}, \varsigma_{k}\right) \\
& =\sum_{k=1}^{K}\left(-\lambda_{k}\right)+\sum_{k=1}^{K} \sum_{m=1}^{M} \xi_{k, m}\left(\frac{2_{k}^{\widehat{R}_{k, m}}-1}{\beta_{k, m}}-P_{\max }\right)+\varphi\left(\sum_{k=1}^{K} \sum_{m=1}^{M} \frac{2^{\hat{R}_{k, m}}-1}{\beta_{k, m}}-P_{0}\right) \\
& +\sum_{m=1}^{M} \psi_{m}\left(\sum_{k=1}^{K} L_{k, m}-1\right)+\sum_{k=1}^{K} \sum_{m=1}^{M} \phi_{k, m}\left(L_{k, m}-1\right)-\sum_{k=1}^{K} \sum_{m=1}^{M} v_{k, m} L_{k, m} \\
& +\sum_{k=1}^{K} \sum_{m=1}^{M} \zeta_{k, m}\left(\widehat{R}_{k, m} \Delta f-R_{\max }\right)-\sum_{k=1}^{K} \sum_{m=1}^{M} \varepsilon_{k, m} \widehat{R}_{k, m} \Delta f \\
& +\sum_{k=1}^{K} \eta_{k}\left(r_{k}^{\text {Req }}+\lambda_{k}-\sum_{m=1}^{M} \widehat{R}_{k, m} \Delta f L_{k, m}\right)-\sum_{k=1}^{K} \zeta_{k} \lambda_{k}
\end{aligned}
$$

where,

$\xi_{k, m}, \varphi, \psi_{m}, \phi_{k, m}, \nu_{k, m}, \zeta_{k, m}, \varepsilon_{k, m}, \eta_{k}, \varsigma_{k}, k \in \mathcal{K}, m \in \mathcal{M}$ are the Lagrange multipliers.

Let

$\widehat{R}_{k, m^{\prime}}^{*} L_{k, m^{\prime}}^{*} \lambda_{k,}^{*} \xi_{k, m^{\prime}}^{*} \varphi^{*}, \psi_{m^{\prime}}^{*} \phi_{k, m^{\prime}}^{*} v_{k, m^{\prime}}^{*} \zeta_{k, m^{\prime}}^{*} \varepsilon_{k, m^{\prime}}^{*} \eta_{k^{\prime}}^{*} \zeta_{k}^{*}, \quad$ be an optimal solution set, then the KKT conditions state that [19]:

$$
\begin{aligned}
& \xi_{k, m}^{*} \geq 0, \varphi^{*} \geq 0, \psi_{m}^{*} \geq 0, \phi_{k, m}^{*} \geq 0, v_{k, m}^{*} \geq 0, \\
& \zeta_{k, m}^{*} \geq 0, \varepsilon_{k, m}^{*} \geq 0, \eta_{k}^{*} \geq 0, \varsigma_{k}^{*} \geq 0 \\
& \xi_{k, m}^{*}\left(\frac{2^{\widehat{R}_{k, m}^{*}}-1}{\beta_{k, m}}-P_{\max }\right)=0 \\
& \varphi^{*}\left(\sum_{k=1}^{K} \sum_{m=1}^{M} \frac{2^{\widehat{R}_{k, m}^{*}}-1}{\beta_{k, m}}-P_{0}\right)=0 \\
& \psi_{m}^{*}\left(\sum_{k=1}^{K} L_{k, m}^{*}-1\right)=0 \\
& \phi_{k, m}^{*}\left(L_{k, m}^{*}-1\right)=0, \quad-v_{k, m}^{*} L_{k, m}^{*}=0 \\
& \zeta_{k, m}^{*}\left(\widehat{R}_{k, m}^{*} \Delta f-R_{\max }\right)=0, \varepsilon_{k, m}^{*} \widehat{R}_{k, m}^{*} \Delta f=0 \\
& \eta_{k}^{*}\left(r_{k}^{\mathrm{Req}}+\lambda_{k}^{*}-\sum_{m=1}^{M} \widehat{R}_{k, m}^{*} \Delta f L_{k, m}^{*}\right)=0 \\
& \zeta_{k}^{*} \lambda_{k}^{*}=0 \\
& \left(\xi_{k, m}^{*}+\varphi^{*}\right) \frac{2^{\widehat{R}_{k, m}^{*}} \ln (2)}{\Delta f \beta_{k, m}}-\eta_{k}^{*} L_{k, m}^{*}=\varepsilon_{k, m}^{*}-\zeta_{k, m}^{*}
\end{aligned}
$$

$$
\begin{aligned}
& \psi_{m}^{*}+\phi_{k, m}^{*}-v_{k, m}^{*}-\eta_{k}^{*} \widehat{R}_{k, m}^{*} \Delta f=0 \\
& -1+\eta_{k}^{*}-\varsigma_{k}^{*}=0
\end{aligned}
$$

Equations (76)-(78) are obtained by setting $\partial L / \partial \widehat{R}_{k, m}=0, \quad \partial L / \partial L_{k, m}=0, \quad$ and $\partial L / \partial \lambda_{k}=0$ respectively.

In order to analyze the KKT consitions and get the optimal solution, we have the following steps to solve Problem OP-B:

(1) Step 1: Power allocation

From (76), the following equation can be obtained:

$$
2^{\widehat{R}_{k, m}^{*}} \frac{\ln (2)}{\Delta f \beta_{k, m}}-\frac{\eta_{k}^{*} L_{k, m}^{*}}{\xi_{k, m}^{*}+\varphi^{*}}=\frac{\varepsilon_{k, m}^{*}-\zeta_{k, m}^{*}}{\xi_{k, m}^{*}+\varphi^{*}}
$$

According to (68) and (73), we know that $\zeta_{k, m}^{*}$ and $\varepsilon_{k, m}^{*}$ cannot be both positive and they are all nonnegative. So the optimal values of $\zeta_{k, m}^{*}$ and $\varepsilon_{k, m}^{*}$ can only be one of the following cases:

$$
\begin{aligned}
& \varepsilon_{k, m}^{*}>0, \zeta_{k, m}^{*}=0 \\
& \varepsilon_{k, m}^{*}=0, \zeta_{k, m}^{*}>0 \\
& \varepsilon_{k, m}^{*}=0, \zeta_{k, m}^{*}=0
\end{aligned}
$$

Here, we set $\omega_{k}=\frac{\eta_{k}^{*} L_{k, m}^{*}}{\xi_{k, m}^{*}+\varphi^{*}}$, therefore, if $\omega_{k}<2^{\widehat{R}_{k, m}^{*}} \frac{\ln (2)}{\Delta f \beta_{k, m}}$, that means we must have $\varepsilon_{k, m}^{*}>0, \zeta_{k, m}^{*}=0$. So, according to (73), we must have $\widehat{R}_{k, m}^{*}=0$.

If $2^{\widehat{R}_{k, m}^{*}} \frac{\ln (2)}{\Delta f \beta_{k, m}} \leq \omega_{k} \leq 2^{\widehat{R}_{\max }} \frac{\ln (2)}{\Delta f \beta_{k, m}}$, that means $0 \leq \widehat{R}_{k, m}^{*} \leq \widehat{R}_{\max }$, where, $\widehat{R}_{\max }=\frac{R_{\max }}{\Delta f}$, we must have $\varepsilon_{k, m}^{*}=0, \zeta_{k, m}^{*}=0$. So, according to (79), we can get that $\widehat{R}_{k, m}^{*}=\log _{2}\left(\frac{\omega_{k} \Delta f \beta_{k, m}}{\ln (2)}\right)$.

If $\omega_{k}>2^{\widehat{R}_{\max }} \frac{\ln (2)}{\Delta f \beta_{k, m}}$, we must have $\varepsilon_{k, m}^{*}=0, \zeta_{k, m}^{*}>0$.

According to (76), we must have $\widehat{R}_{k, m}^{*}=\widehat{R}_{\max }$.

Therefore, to summarize, the optimal value of $\widehat{R}_{k, m}^{*}$ is:

$$
\widehat{R}_{k, m}^{*}= \begin{cases}0, & \omega_{k}<\frac{\ln (2)}{\Delta f \beta_{k, m}} \\ \log _{2}\left(\frac{\omega_{k} \Delta f \beta_{k, m}}{\ln (2)}\right), & \frac{\ln (2)}{\Delta f \beta_{k, m}} \leq \omega_{k} \leq \frac{2^{\widehat{R}_{\max }} \ln (2)}{\Delta f \beta_{k, m}} \\ \widehat{R}_{\max }, & \omega_{k}>\frac{2^{\widehat{R}_{\max }} \ln (2)}{\Delta f \beta_{k, m}}\end{cases}
$$


The following optimal power allocation $P_{k, m}^{*}=\frac{2^{\widehat{R}_{m a x}^{*}}-1}{\beta_{k, m}}$ can be obtained:

$$
P_{k, m}^{*}= \begin{cases}0, & \omega_{k}<\frac{\ln (2)}{\Delta f \beta_{k, m}} \\ \frac{\omega_{k} \Delta f}{\ln (2)}-\frac{1}{\beta_{k, m}}, & \frac{\ln (2)}{\Delta f \beta_{k, m}} \leq \omega_{k} \leq \frac{2^{\widehat{R}_{\max }} \ln (2)}{\Delta f \beta_{k, m}} \\ \frac{2^{\widehat{R}_{\max }}-1}{\beta_{k, m}}, & \omega_{k}>\frac{2^{\widehat{R}_{\max }} \ln (2)}{\Delta f \beta_{k, m}}\end{cases}
$$

where $\omega_{k}=\frac{\eta_{k}^{*} L_{k, m}^{*}}{\xi_{k, m}^{*}+\varphi^{*}}$, represents the update of the multipliers. Moreover, $\omega_{k}$ is viewed as the IWF level for the SU $k$ and will be discussed later.

\section{(2) Step 2: Subcarrier allocation}

For simplicity and in order to maximize the system throughput, $L_{k, m}^{*}$ is set to be either 0 or 1 , and one subcarrier must be allocated to any user. So, for any given time slot $t$ and subcarrier $m$, there is only one SU $k^{*}$ with a nonzero value of $L_{k^{*}, m}^{*}$ and $L_{k^{*}, m}^{*}=1$ according to (64). Now, we'll discuss how to determine the SU $k^{*}$.

For SU $k^{*}$, according to (72), we have $\phi_{k^{*}, m}^{*} \geq 0$, and $v_{k^{*}, m}^{*}=0$, and it follows from (77) that

$$
\psi_{m}^{*}=\eta_{k^{*}}^{*} \widehat{R}_{k^{*}, m}^{*} \Delta f-\phi_{k^{*}, m}^{*}
$$

For any other SU $k \neq k^{*}, L_{k, m}^{*}=0$, according to (72), we have $\phi_{k, m}^{*}=0$, and $v_{k, m}^{*} \geq 0$, combined with (77), we have

$$
\psi_{m}^{*}=v_{k, m}^{*}+\eta_{k}^{*} \widehat{R}_{k, m}^{*} \Delta f
$$

Compare (82) with (83), we have

$$
\eta_{k^{*}}^{*} \widehat{R}_{k^{*}, m}^{*} \geq \eta_{k}^{*} \widehat{R}_{k, m}^{*}
$$

The subcarrier allocation strategy for any subcarrier $m$ is

$$
\begin{aligned}
& L_{k, m}^{*}=\left\{\begin{array}{l}
1, k=k^{*} \text { and } m \in \mathcal{M} \\
0, \text { otherwise }
\end{array}\right. \\
& k^{*}=\arg \max \quad \eta_{k}^{*} \widehat{R}_{k, m}^{*}
\end{aligned}
$$

where $\widehat{R}_{k, m}^{*}$ is the optimal value from (80). Suppose that for a given $t$ and $m$, the values of $\eta_{k}^{*} \widehat{R}_{k, m}^{*}$ are the same for several users, we will choose one SU arbitrarily.

(3) Step 3: IWF level

For all SUs, according to KKT conditions, when $\lambda_{k}^{*}>0$, according to (75), $\varsigma_{k}^{*}=0$ and according to (78), $\eta_{k}^{*}=1$, so $\omega_{k}=\frac{L_{k, m}^{*}}{\xi_{k, m}^{*}+\varphi^{*}}$. When $\lambda_{k}^{*}=0$, then $\varsigma_{k}^{*} \geq 0$ and $\eta_{k}^{*}=1+\varsigma_{k}^{*}$, so $\omega_{k}=\frac{\left(1+\varsigma_{k}^{*}\right) L_{k, m}^{*}}{\xi_{k, m}^{*}+\varphi^{*}}$. The initial value of
$\omega_{B}=\frac{1}{\xi_{k, m}^{*}+\varphi^{*}}$ in $\omega_{k}$ can be set to 1 . Let $\omega_{B}=\frac{1}{\xi_{k, m}^{*}+\varphi^{*}}$ be the base water-level for all SUs.

From (68)-(70), if the optimal value $\phi^{*}>0$, that means all the power has been used to optimize the system throughput. Therefore, the initial value of $\phi^{*}$ and $\xi_{k, m}^{*}$ should be set to $\phi^{*}>0$ and $\xi_{k, m}^{*}>0$ to obtain the maximum system throughput. Therefore, to summarize, the water-filling level $\omega_{k}$ is:

$$
\omega_{k}= \begin{cases}\omega_{B}, & \lambda_{k}^{*}>0 \\ \left(1+\zeta_{k}^{*}\right) \omega_{B}, & \lambda_{k}^{*}=0\end{cases}
$$

where $\omega_{B}=1 /\left(\xi_{k, m}^{*}+\varphi^{*}\right), \xi_{k, m}^{*}>0, \varphi^{*}>0$.

\section{Competing interests}

The authors declare that they have no competing interests.

\section{Received: 29 April 2011 Accepted: 9 February 2012}

Published: 9 February 2012

\section{References}

1. Federal Communications Commission, FCC Spectrum policy task force report, Report of the Spectrum Efficiency Working Group. ET Docket no. 02-135 (Nov 2002)

2. J Mitola III, Cognitive radio: An integrated agent architecture for software defined radio, Ph.D. dissertation (Royal Institute of Technology (KTH), Stockholm, Swedem, 2000)

3. S Haykin, Cognitive radios: brain-empowered wireless communications. IEEE J Sel Areas Commun. 23(2), 13-18 (2005)

4. TA Weiss, FK Jondral, Spectrum pooling: an innovative strategy for the enhancement of spectrum efficiency. IEEE Commun Mag. 42(3), S8-14 (2004)

5. T Qin, C Leung, Fair adaptive resource allocation for multiuser OFDM cognitive radio systems, in Proc 2nd Int Conf CHINACOM, Shanghai, China, 115-119 (Aug 2007)

6. A Attar, O Holland, MR Nakhai, AH Aghvami, Interference limited resource allocation for cognitive radio in orthogonal frequency-division multiplexing networks. IET Commun. 2(6), 806-814 (2008). doi:10.1049/iet-com:20070355

7. H Su, X Zhang, Cross-layer-based opportunistic MAC protocols for QoS provisionings over cognitive radio wireless networks. IEEE J Sel Areas Commun. 26(1), 118-129 (2008)

8. JW Mwangoka, KB Letaief, Z Cao, Robust end-to-end QoS maintenance in non-contiguous OFDM based cognitive radios, in Proc IEEE ICC, Beijing, China, 2905-2909 (May 2008)

9. R Wang, VKN Lau, L LV, B Chen, Joint cross-layer scheduling and spectrum sensing for OFDMA cognitive radio systems. IEEE Trans Wirel Commun. 8(5), 2410-2416 (2009)

10. $Y$ Zhang, C Leung, Cross-layer resource allocation for mixed services in multiuser OFDM-based cognitive radio systems. IEEE Trans Veh Technol. 58(8), 4605-4619 (2009)

11. TA Weiss, J Hillenbrand, A Krohn, FK Jondral, Mutual interference in OFDMbased spectrum pooling systems, Proc of IEEE 59th Vehicular Technology Conference (VTC 2004-Spring), Milan, Italy, 1873-1877 4, (2004)

12. W Ren, Q Zhao, A Swami, Power control in cognitive radio networks: how to cross a multi-lane highway. IEEE J Sel Areas Commun. 27(7), 1283-1296 (2009)

13. G Zhao, GY Li, C Yang, Proactive detection of spectrum opportunities in primary systems with power control. IEEE Trans Wirel Commun. 8(9), 4815-4823 (2009)

14. A Goldsmith, Wireless Communications, (Cambridge University Press, Cambridge, 2005)

15. H Celebi, H Arslan, Cognitive positioning systems. IEEE Trans Wirel Commun. 6(12), 4475-4483 (2007)

16. H Nam, MB Ghorbel, MS Alouini, Location-based resource allocation for OFDMA cognitive radio systems, in Proc CrownCom 2010, Cannes, France, 1-5 (9-11 June 2010) 
17. M Ben Ghorbel, H Nam, MS Alouini, Discrete rate resource allocation for OFDMA cognitive radio systems with location information, in PIMRC workshops 2010, Instanbul, Turkey, 247-251 (Sep 2010)

18. Y Zhang, Resource allocation for OFDM-based cognitive radio systems, Ph D. dissertation (Univ. British Columbia, Vancouver, BC, Canada, 2008)

19. S Boyd, L Vandenberghe, Convex Optimization, (Cambridge University Press, Cambridge, 2004)

20. DG Luenberger, Optimization by Vector Space Methods, Wiley, NewYork, (1969)

21. K Kim, Y Han, S-L Kim, Joint subcarrier and power allocation in uplink OFDMA systems. IEEE Commun Lett. 9, 526-528 (2005). doi:10.1109/ LCOMM.2005.1437359

22. Y Ma, Rate maximization for downlink OFDMA with proportional fairness. IEEE Trans Veh Technol. 57, 3267-3274 (2008)

doi:10.1186/1687-1499-2012-41

Cite this article as: Peng and Fujii: Joint cross-layer resource allocation and interference avoidance with QoS support for cognitive radio systems. EURASIP Journal on Wireless Communications and Networking 2012 2012:41.

\section{Submit your manuscript to a SpringerOpen ${ }^{\circ}$ journal and benefit from:}

- Convenient online submission

- Rigorous peer review

- Immediate publication on acceptance

- Open access: articles freely available online

- High visibility within the field

- Retaining the copyright to your article

Submit your next manuscript at $\gg$ springeropen.com 